Pacific Journal of Mathematics

CONSTRUCTIONS OF TWOFOLD BRANCHED COVERING 


\title{
CONSTRUCTIONS OF TWO-FOLD BRANCHED COVERING SPACES
}

\author{
JosE M. Montesinos AND Wilbur Whitten
}

To Deane Montgomery

\begin{abstract}
By equivariantly pasting together exteriors of links in $S^{3}$ that are invariant under several different involutions of $S^{3}$, we construct closed orientable 3-manifolds that are two-fold branched covering spaces of $S^{3}$ in distinct ways, that is, with different branch sets. Sufficient conditions are given to guarantee when the constructed manifold $M$ admits an induced involution, $h$, and when $M / h \cong S^{3}$. Using the theory of characteristic submanifolds for Haken manifolds with incompressible boundary components, we also prove that doubles, $D(K, \rho)$, of prime knots that are not strongly invertible are characterized by their two-fold branched covering spaces, when $\rho \neq 0$. If, however, $K$ is strongly invertible, then the manifold branch covers distinct knots. Finally, the authors characterize the type of a prime knot by the double covers of the doubled knots, $D(K ; \rho, \eta)$ and $D\left(K^{*} ; \rho, \eta\right)$, of $K$ and its mirror image $K^{*}$ when $\rho$ and $\eta$ are fixed, with $\rho \neq 0$ and $\eta \in\{-2,2\}$.
\end{abstract}

With each two-fold branched covering map, $p: M^{3} \rightarrow N^{3}$, there is associated a PL involution, $h: M \rightarrow M$, that induces $p$. There can, however, be other PL involutions on $M$ that are not equivalent to $h$, but nevertheless are covering involutions for two-fold branched covering maps of $M$ (cf. [BGM]). Our purpose, in this paper, is to introduce ways of detecting such involutions and controlling their number. We begin with compact 3-manifolds with several obvious PL involutions.

An oriented link $\tilde{L}$ in $M$ is 2-symmetric, if $N^{3} \cong S^{3}$ and if $h(\tilde{L})=\tilde{L}$. In $§ 1$, we give examples of knots and links in $S^{3}$ that are 2-symmetric in two or more ways; for example, a trefoil knot is both strongly invertible and periodic (definitions in $\$ 1$ ). In $\$ 2$, we paste the exteriors, $E(\tilde{L})$ and $E\left(\tilde{L}^{\prime}\right)$, of 2-symmetric links, $\tilde{L}$ and $\tilde{L}^{\prime}$, together along a torus-boundary component of each exterior; Proposition 2.1 gives the pasting instructions $f$ that must be followed in order for the involutions, $h$ and $h^{\prime}$, of $E(\tilde{L})$ and $E\left(\tilde{L}^{\prime}\right)$ to extend to an involution $h_{f}$ of $E(\tilde{L}) \cup_{f} E\left(\tilde{L}^{\prime}\right)$. Theorems 2.2 and 2.3 allow us to conclude, under fairly relaxed conditions, that the orbit space of $h_{f}$ is $S^{3}$. 
In the remainder of $\S 2$, we apply these results to our examples of 2-symmetric links to construct two-fold branched covering spaces of $S^{3}$. (Recall that any such branched covering space can always be constructed by suitable surgery on some strongly invertible link in $S^{3}$; this idea was first introduced in $\left[\mathbf{M o}_{\mathbf{1}}\right]$.) The construction process shows clearly that many of the manifolds produced have at least two different Heegaard splittings. Some of our covering spaces are hyperbolic manifolds (obtained by surgery on the figure-eight knot) with different Heegaard splittings; W. Jaco has asked whether a hyperbolic manifold could have this property.

In $\S 3$, we take the opposite tack and construct 3-manifolds that are two-fold branched covering spaces of $S^{3}$ in exactly one way; that is, these manifolds characterize the type of the link in $S^{3}$ over which they branch. Theorem 3.7 characterizes many doubled-knot types in this way. If, however, $K$ is a prime, strongly invertible knot, then the two-fold branched covering space of any double of $K$ covers $S^{3}$ in at least two distinct ways (Proposition 3.8). Along the way, we classify the strong equivalence classes of involutions on Seifert fibered manifolds of the form $(\mathrm{O}, \mathrm{o} ; 0 \mid 0$; $2 k / 1$ ) with fibered neighborhoods of two regular fibers removed, for $k$ a nonzero integer (Theorem 3.6). In Corollary 3.10, we characterize the knot type of a prime knot by the topological type of the two-fold branched covering space of a certain doubled knot.

We wish to thank S. Bleiler, W. B. R. Lickorish, J. Przytycki and M. Sakuma for helpful comments. We work in the PL category. One can refer to [Ja] for most basic definitions and results.

1. Examples of 2-symmetric links. Let $p: M \rightarrow S^{3}$ be a two-fold branched covering map, let $h$ be the covering involution, and let $B$ be the branch set of $p$. An oriented link $\tilde{L}$ in $M$ is 2 -symmetric if $h(\tilde{L})=\tilde{L}$. The image $L(=p \tilde{L})$ is a disjoint union of knots and arcs. The preimage of an arc is a strongly invertible subknot of $\tilde{L}$; that of a knot in $L \cap B$, an invariant subknot of $\tilde{L}$; that of a component $K$ of $L$ such that $\operatorname{Lk}(B, K) \equiv 1$ (mod 2), a periodic subknot of $\tilde{L}$. The link $\tilde{L}$ is periodic, if all components of $\tilde{L}$ are periodic simultaneously. Finally, the preimage of a component $K$ of $L$ such that $\operatorname{Lk}(B, K) \equiv 0(\bmod 2)$ is an interchangeable two-component sublink of $\tilde{L}$.

Let $U$ be a relative regular neighborhood of $L$ in $\left(S^{3}, B\right)$. In each torus component of $\partial U$, we take a meridian-longitude pair $(m, l)$ of the corresponding knot. In each spherical component of $\partial U$, we take a pair ( $m, l$ ) of arcs such that $m \cap B=\partial m$ and $l \cap B=\partial l$; moreover, $m \cap l$ is one point, and $m$ together with an arc of $U \cap B$ bound a disk in $U$. In each component of the preimage of $\partial U$, take a coordinate pair $(\tilde{m}, \tilde{l})$ that 
projects under $p$ onto one of the above defined pairs $(m, l)$. These coordinate pairs are related to the canonical meridian-longitude pairs of the components of $\tilde{L}$ as follows.

Proposition 1.1. Assume that $M=S^{3}$. Then the coordinate pair ( $\tilde{m}, \tilde{l})$ of an invariant or periodic subknot $\tilde{K}$ of $\tilde{L}$ is also a meridian-longitude pair of $\tilde{K}$. If $(\tilde{m}, \tilde{l})$ belongs to a component $\tilde{K}_{1}$ of an interchangeable sublink $\tilde{K}_{1} \cup \tilde{K}_{2}$ of $\tilde{L}$, then $\tilde{m}$ is a meridian of $\tilde{K}_{1}$, and any longitude of $\tilde{K}_{1}$ is homologous to $\tilde{l}+\operatorname{Lk}\left(\tilde{K}_{1}, \tilde{K}_{2}\right) \tilde{m}$ in $p^{-1}(\partial U)$.

Proof. Let $A$ be an oriented, connected 2-manifold bounded by $K$ and transverse to $B$. Then $\tilde{A}\left(=p^{-1} A\right)$ is an oriented 2-manifold and $\partial \tilde{A}=\tilde{K}$. Assume that $l$ is contained in $A$. If $\tilde{K}\left(=p^{-1} K\right)$ is connected, then $\tilde{l}$ is contained in $\tilde{A}$ and is a longitude of $\tilde{K}$. If $\tilde{K}$ is not connected, then the canonical longitude $\tilde{l}^{\prime}$ of $\tilde{K}_{1}$ is homologous to $\tilde{l}+\operatorname{Int}\left(\tilde{A}, \tilde{l}^{\prime}\right) \tilde{m}$ in $p^{-1}(\partial U)$. The second part of the proposition follows, since

$$
\operatorname{Int}\left(\tilde{A}, \tilde{l}^{\prime}\right)=\operatorname{Lk}\left(\tilde{K}_{1}, \tilde{l}^{\prime}\right)+\operatorname{Lk}\left(\tilde{K}_{2}, \tilde{l}^{\prime}\right)=\operatorname{Lk}\left(\tilde{K}_{1}, \tilde{K}_{2}\right) \text {. }
$$

REMARK. When $M=S^{3}$, we shall take the $\operatorname{arcs}(m, l)$ of any spherical component of $\partial U$ so that the preimages $(\tilde{m}, \tilde{l})$ form a meridian-longitude pair of the corresponding strongly invertible subknot of $\tilde{L}$.

We are interested in links in $S^{3}$ with more than one interpretation as a 2-symmetric link. We give some examples.

(a) A strongly invertible link that is interchangeable in two different ways.
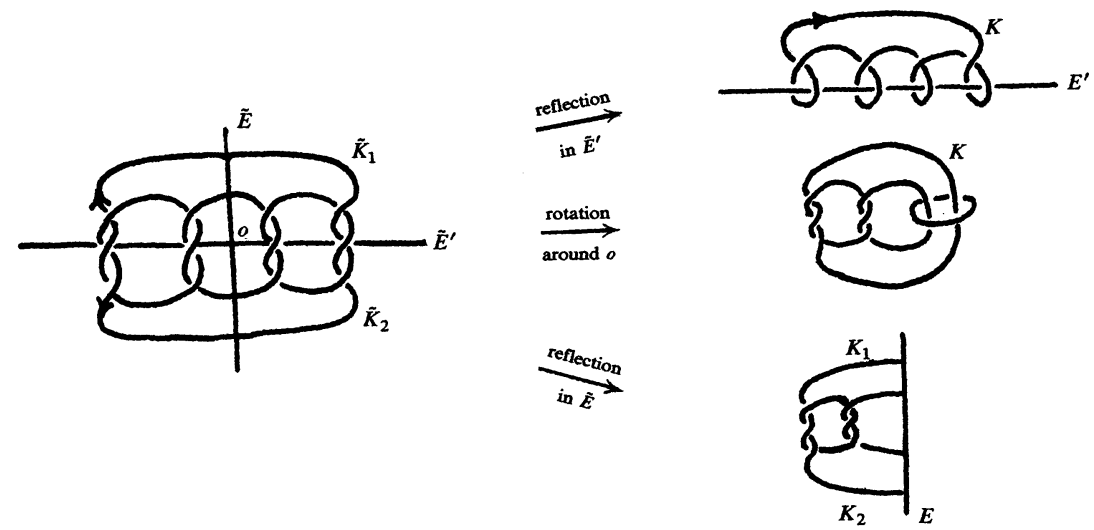

FigURE 1 
(b) A periodic link that is interchangeable in two different ways.

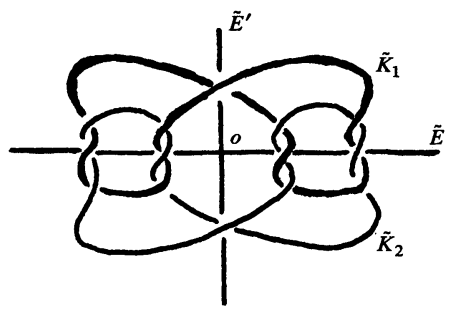

$$
\begin{aligned}
& \underset{\text { in } \tilde{E}}{\stackrel{\text { reflection }}{\longrightarrow}} \\
& \underset{\text { around } o}{\text { rotation }} \\
& \underset{\text { in } \tilde{E}^{\prime}}{\text { reflection }}
\end{aligned}
$$
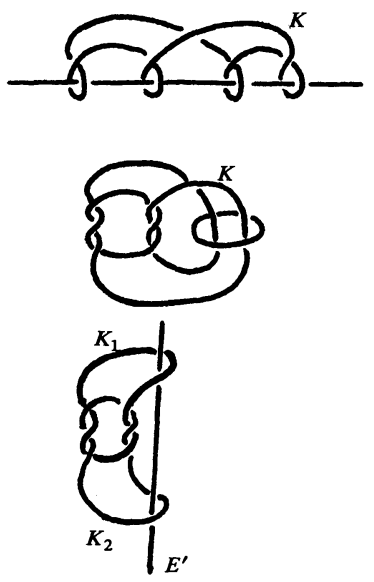

FIGURE 2

(c) A knot that is periodic and strongly invertible.

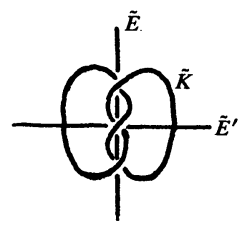

$$
\underset{\text { around } \tilde{E}}{\text { rotation }}
$$

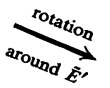

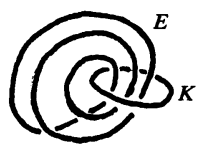

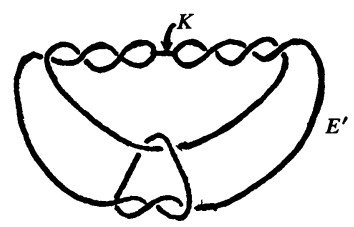

Figure 3

REMARK. In Figure 3 (and in the rest of the figures of this section), we depict the arc $K$ so that its coordinates $(m, l)$ lie in the plane of projection. We note that the two constructions of Figure 3 first appeared in $\left[\mathbf{M o}_{2}\right]$. 
(d) An amphicheiral knot: periodic and, in two ways, strongly invertible.
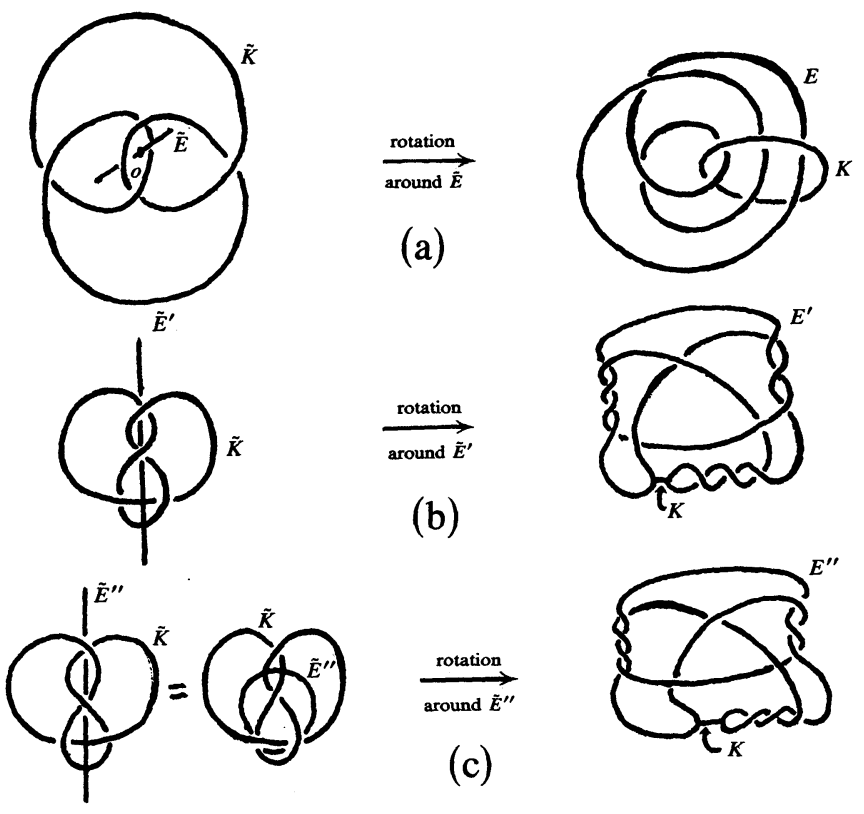

$\overrightarrow{\text { around } \tilde{E}^{\prime}}$

(b)
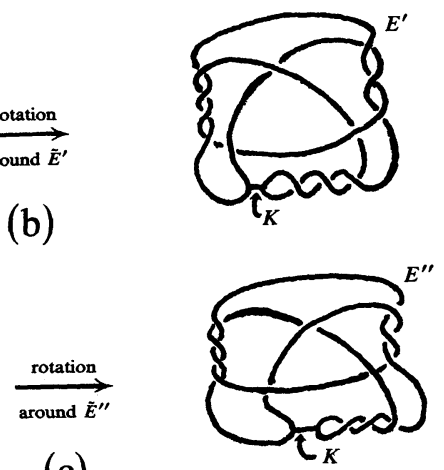

(c)

FIGURE 4

(e) $K \sharp K$ : strongly invertible in two different ways.
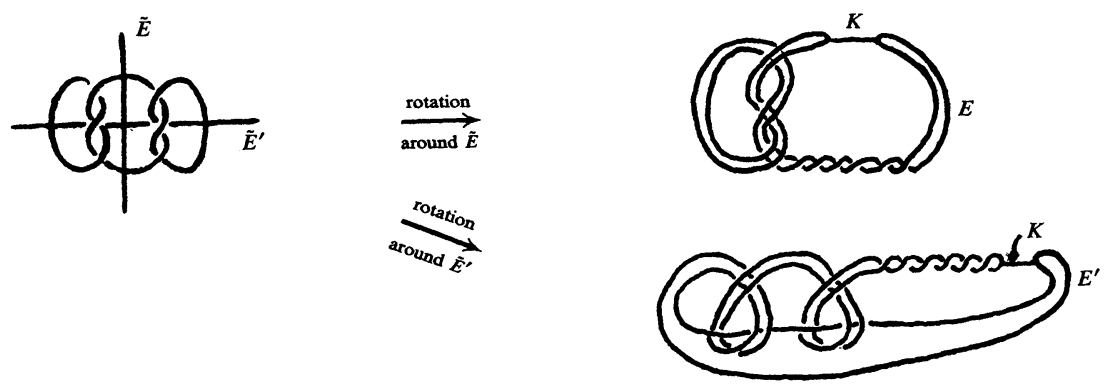
(f) A knot that is invariant, periodic, and strongly invertible.

First Example.
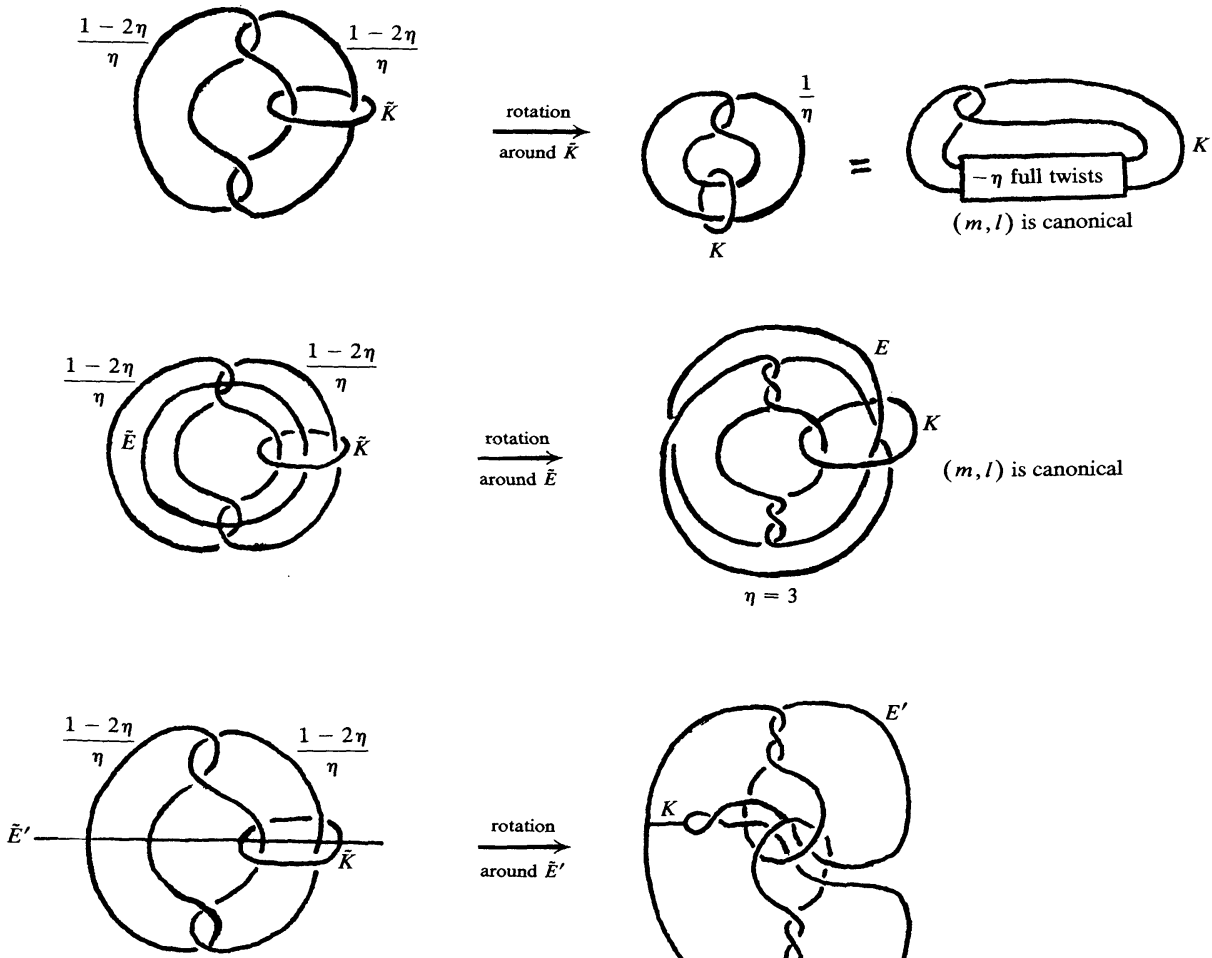

$\underset{\text { around } \tilde{E}}{\stackrel{\text { rotation }}{\longrightarrow}}$

$\underset{\text { around } \tilde{E}^{\prime}}{\stackrel{\text { rotation }}{\longrightarrow}}$

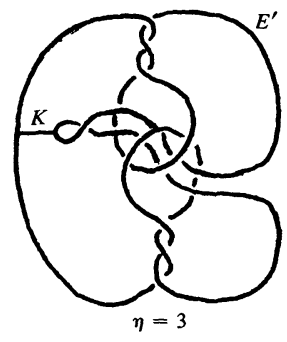

FIGURE 6

The knot $\tilde{K}$ (see Figure 6 ) is contained in the manifold $M$ obtained by surgery on a link $N$ of two components. We take $(\tilde{m}, \tilde{l})$ as a meridianlongitude pair of $\tilde{K}$ thought of as part of the link, $\tilde{K} \cup N \subset S^{3}$.

REMARK. For $\eta=1$, the manifold $M$ is the lens space $L(3,1)$ depicted in Figure 7, which is a two-fold covering space of $S^{3}$ branched over the trefoil.

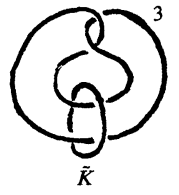

Figure 7 
Second Example.
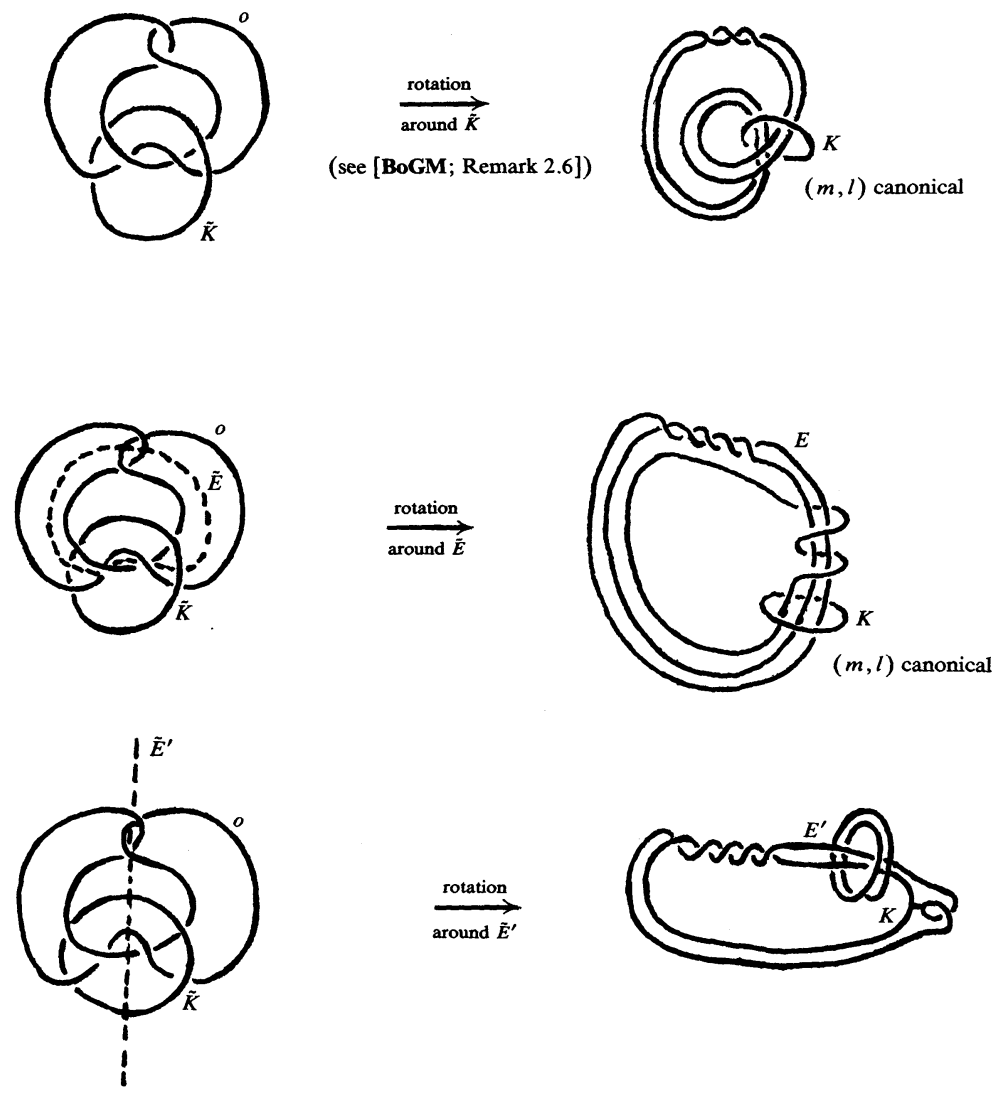

Figure 8

The knot $\tilde{K}$ is contained in the manifold $M$ obtained by surgery on the figure-eight $\operatorname{knot} N$. We take $(\tilde{m}, \tilde{l})$ as a meridian-longitude pair of $\tilde{K}$ thought of as part of the link $\tilde{K} \cup N$.

2. Constructions of two-fold branched coverings of $\mathbf{S}^{\mathbf{3}}$. Our goal in this section is to construct two-fold branched covering spaces of $S^{3}$ by pasting together the exterior of links that are 2-symmetric in different ways. The following proposition gives the condition under which the resulting manifold admits an induced involution.

Proposition 2.1. Let $E(\tilde{L})$ and $E\left(\tilde{L}^{\prime}\right)$ denote the exteriors of 2-symmetric links $\tilde{L}$ and $\tilde{L}^{\prime}$ in the manifolds $M$ and $M^{\prime}$, with involutions $h$ and $h^{\prime}$. Let $\tilde{T}$ and $\tilde{T}^{\prime}$ be tori in $\partial E(\tilde{L})$ and $\partial E\left(\tilde{L}^{\prime}\right)$ with coordinates $(\tilde{m}, \tilde{l})$ and 
$\left(\tilde{m}^{\prime}, \tilde{l}^{\prime}\right)$. Form $E(\tilde{L}) \cup \tilde{f} E\left(\tilde{L}^{\prime}\right)$, that is, the result of pasting $E(\tilde{L})$ and $E\left(\tilde{L}^{\prime}\right)$ together along $\tilde{T}$ and $\tilde{T}^{\prime}$ according to a homeomorphism $\tilde{f}: \tilde{T}^{\prime} \rightarrow \tilde{T}$, such that

$$
(\tilde{\boldsymbol{m}}, \tilde{l})\left(\begin{array}{ll}
\boldsymbol{\alpha} & \gamma \\
\boldsymbol{\beta} & \delta
\end{array}\right) \sim\left(\tilde{f} \tilde{m}^{\prime}, \tilde{f l^{\prime}}\right)
$$

on $\tilde{T} ;$ the matrix, $\left(\begin{array}{ll}\alpha & \gamma \\ \beta & \gamma\end{array}\right)$ is an integral matrix with determinant -1 . Then the involutions $h$ and $h^{\prime}$ extend to an involution of $E(\tilde{L}) \cup_{\tilde{f}} E\left(\tilde{L}^{\prime}\right)$ if and only if one of the following holds:

(i) both $\tilde{T}$ and $\tilde{T}^{\prime}$ correspond to periodic knots and $\gamma$ is even;

(ii) $\tilde{T}$ is periodic, $\tilde{T}^{\prime}$ is invariant, and $\alpha$ is even;

(iii) $\tilde{T}$ is invariant, $\tilde{T}^{\prime}$ is periodic, and $\delta$ is even;

(iv) both $\tilde{T}$ and $\tilde{T}^{\prime}$ are invariant, and $\beta$ is even; or

(v) both $\tilde{T}$ and $\tilde{T}^{\prime}$ are strongly invertible.

It is more difficult to decide whether the orbit space under such involutions (as in Proposition 2.1) is $S^{3}$. The next two theorems give two interesting particular cases for which this happens.

THeOREM 2.2. Let $\tilde{L}$ be a 2-symmetric link in $\tilde{M}$ such that the link part of $L$ is trivial, that is, a collection of unlinked trivial knots. For each component $\tilde{K}$ of $\tilde{L}$, let $N(\tilde{K})$ be a knot in a manifold. We assume that $N(\tilde{K})$ is 2-symmetric, except when $\tilde{K}$ belongs to an interchangeable sublink of $\tilde{L}$. Identify the boundaries of $E(\tilde{L})$ and $E(N(\tilde{K}))$ by a homeomorphism, $f: \partial E(N(\tilde{K})) \rightarrow \partial U(\tilde{K})$, given homologically by

$$
(\tilde{m}, \tilde{l})\left(\begin{array}{ll}
\alpha & \gamma \\
\beta & \delta
\end{array}\right) \sim\left(f \tilde{m}^{\prime}, \tilde{f l^{\prime}}\right)
$$

on $\partial U(\tilde{K}) ;$ the $\operatorname{det}\left(\begin{array}{ll}\alpha & \gamma \\ \beta & \delta\end{array}\right)=-1$ and we set $A=\left(\begin{array}{cc}\alpha & \gamma \\ \beta & \delta\end{array}\right)$; the set $U(\tilde{K})$ is a regular neighborhood of $\tilde{K}$ in $\tilde{M}$, the pair $(\tilde{m}, \tilde{l})$ is a coordinate pair for $\tilde{K}$, and $\left(\tilde{m}^{\prime}, \tilde{l}^{\prime}\right)$ is a coordinate pair for $N(\tilde{K})$. Assume also that

(i) if both $\tilde{K}$ and $N(\tilde{K})$ are periodic, then the orbit space of $E(N(\tilde{K}))$ is a solid torus and $A=\left(\begin{array}{ll}\alpha & 2 \\ \beta & \delta\end{array}\right)$;

(ii) if $\tilde{K}$ is periodic and $N(\tilde{K})$ is invariant, then $A=\left(\begin{array}{cc}2 \alpha & 1 \\ \beta & \delta\end{array}\right)$, if the orbit space of $E(N(\tilde{K}))$ is a solid torus, and $A=\left(\begin{array}{ll}0 & 1 \\ 1 & \delta\end{array}\right)$, otherwise;

(iii) if $\tilde{K}$ is invariant and $N(\tilde{K})$ is periodic, then $A=\left(\begin{array}{ll}\alpha & 1 \\ \beta & 2 \delta\end{array}\right)$, if the orbit space of $E(N(\tilde{K}))$ is a solid torus, and $A=\left(\begin{array}{ll}0 & 1 \\ 1 & 2 \delta\end{array}\right)$, otherwise;

(iv) $\tilde{K}$ and $N(\tilde{K})$ are not both invariant;

(v) if $\tilde{K}$ is strongly invertible, then so is $N(\tilde{K})$; and

(vi) if $\tilde{K}$ belongs to an interchangeable sublink $\tilde{K} \cup \tilde{K}^{\prime}$ of $\tilde{L}$, then $N\left(\tilde{K}^{\prime}\right)=N(\tilde{K})$ and both matrices are equal to $\left(\begin{array}{ll}\alpha & 1 \\ \beta & \delta\end{array}\right)$, if $N(\tilde{K})$ is trivial in $S^{3}$, and to $\left(\begin{array}{ll}0 & 1 \\ 1 & \delta\end{array}\right)$, otherwise. 
Then the resulting oriented closed 3-manifold $M$ admits an involution whose orbit space is $S^{3}$.

REMARK. The manifold $M$ of Theorem 2.2(vi) might admit another involution arising from a second involution on $\tilde{M}$ that interchanges $\tilde{K} \cup \tilde{K}^{\prime}$. For example, one involution $h$ on $M$ might arise from an involution on $\tilde{M}$ that preserves the orientation of each component of $\tilde{K} \cup \tilde{K}^{\prime}$, and a second involution $h^{\prime}$ on $M$ might arise from an involution that reverses the orientation of each component of $\tilde{K} \cup \tilde{K}^{\prime}$. In the second case, we must also assume that $N(\tilde{K})\left(=N\left(\tilde{K}^{\prime}\right)\right)$ is invertible, assuming that $M$ itself arises from the first described involution on $\tilde{M}$. Generally, however, $M / h^{\prime} \neq S^{3}$, as example $g(4)$ (following) shows, when $h^{\prime}=h_{C}$ and $h=h_{A}$ in $g(4)$.

EXAMPLES. We have chosen the following examples to illustrate the representation of 3-manifolds as two-fold branched cyclic covering spaces of $S^{3}$ in different ways. We shall use the following notation (Figure 9), in
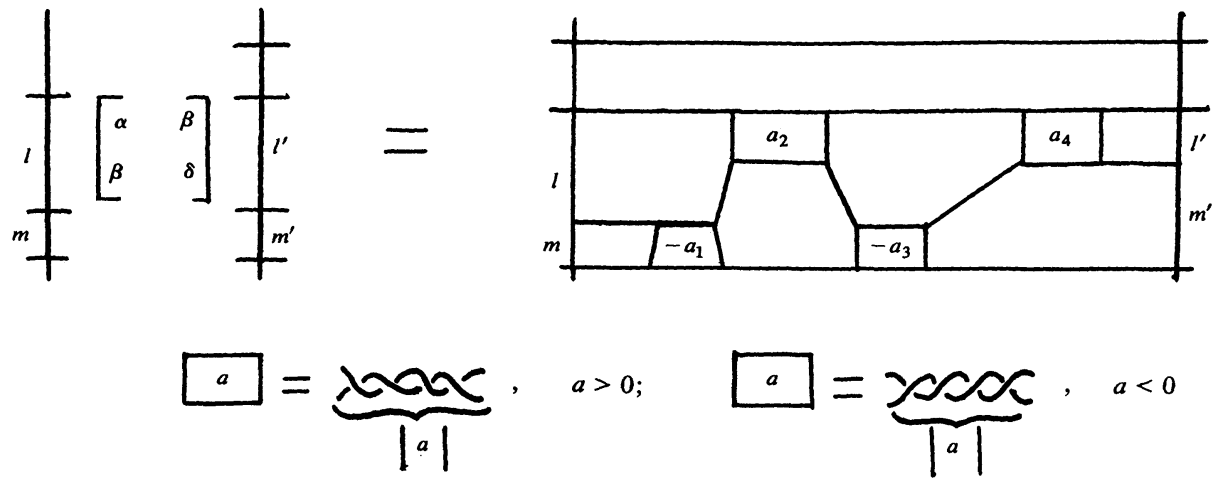

Figure 9

which $\operatorname{det}\left(\begin{array}{ll}\alpha & \gamma \\ \beta & \delta\end{array}\right)=+1$. Set

$$
\left[a_{1}, \ldots, a_{k}\right]=a_{1}+\frac{1}{a_{2}+\cdots+\frac{1}{a_{k}}},
$$

and assume further that $\alpha / \beta=\left[a_{1}, \ldots, a_{2 n}\right]$ and $\gamma / \delta=\left[a_{1}, \ldots, a_{2 n-1}\right]$. Figure 9 corresponds to the case when

$$
\left(\begin{array}{ll}
\alpha & \gamma \\
\beta & \delta
\end{array}\right)=\left(\begin{array}{rr}
43 & 30 \\
10 & 7
\end{array}\right)
$$


We also use the notation of Figure 10 , with $\gamma / \delta=\left[a_{1}, \ldots, a_{2 n-1}\right]$ when $\alpha / \beta=\left[a_{1}, \ldots, a_{2 n}\right]$. In Figure 11, we depict the case $\alpha / \beta=43 / 10$. We now give our examples.

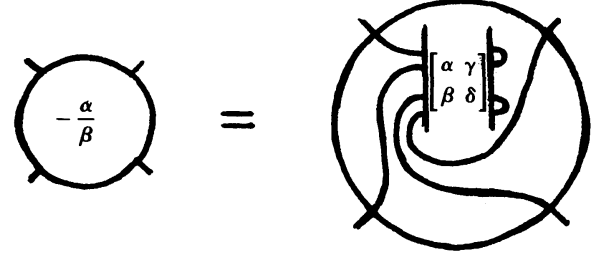

FigURE 10

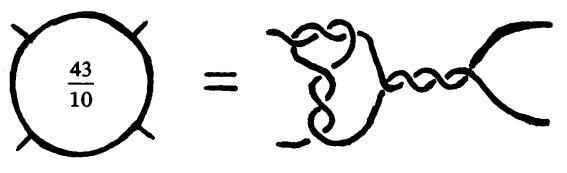

FIGURE 11

(a) Take $\tilde{L}$ to be the link $\tilde{K}_{1} \cup \tilde{K}_{2}$ of Figure 1 , let $N(\tilde{K})$ be a trivial knot in $S^{3}$, and take $A=\left(\begin{array}{ll}0 & 1 \\ 1 & \delta\end{array}\right)$. The manifold $M$ is a two-fold covering space of $S^{3}$ branched over the curves of Figure 12(a), because $\tilde{L}$ is interchangeable, and over the curves of Figure $12(\mathrm{~b})$, because $\tilde{L}$ is strongly invertible.

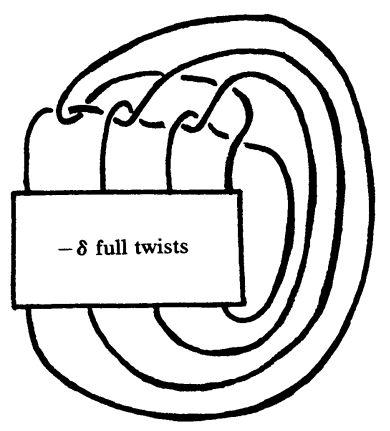

(a)

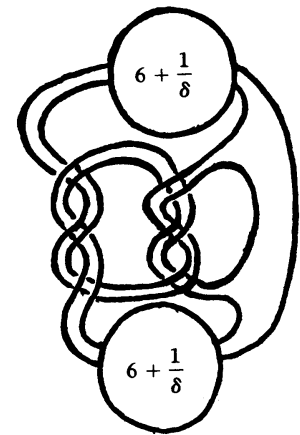

(b)

FIGURE 12

(b) Take $\tilde{L}$ to be the (right-hand) trefoil knot of Figure 3, let $N(\tilde{K})$ be trivial in $S^{3}$, and take $A=\left(\begin{array}{cc}\alpha & 2 \\ \beta & \delta\end{array}\right)$ (respectively, $A=\left(\begin{array}{cc}2 \alpha & 1 \\ \beta & \delta\end{array}\right)$ ). Then the manifold obtained by $2 / \delta$-surgery ( $\delta$ odd) on the trefoil (respectively, $1 / \delta$-surgery) is a two-fold branched covering space of $S^{3}$ in the two ways depicted in Figure 13 (respectively, Figure 14) (cf. [G]). 

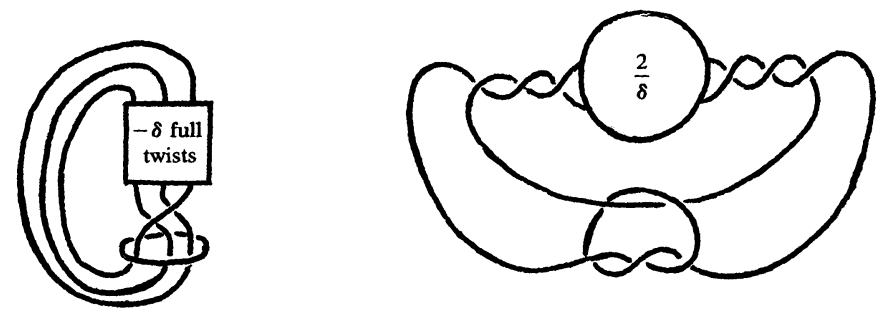

FIGURE 13

REMARK. The knot of Figure $14(\mathrm{a})$ is the $(3,1-6 \delta)$-torus knot; the knot of Figure $14(\mathrm{~b})$, for $\delta=-1$, is the $(-2,3,7)$-pretzel knot (cf. [BGM]). The knots of Figures 14(a) and (b) are equivalent precisely when $\delta=1$. By the same method used in [BGM], the homology 3-spheres obtained by surgery on the trefoil (except the Poincarẽ manifold) have at least two different Heegaard splittings of genus 2 (note that the knot of Figure 12(b) has 3 bridges).
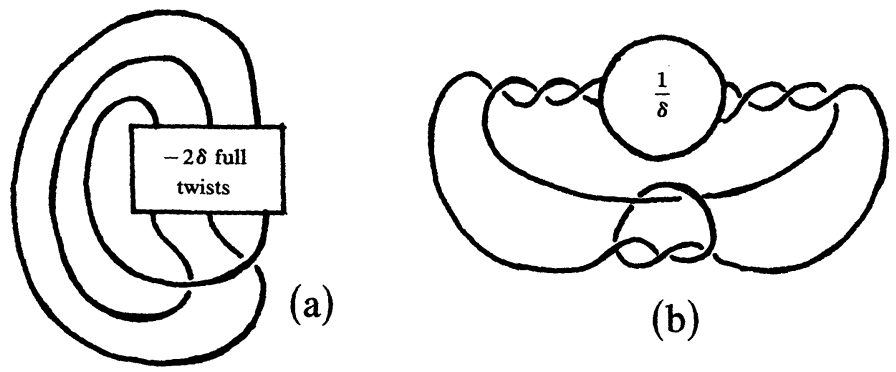

(a)

(b)

Figure 14

(c) Take $\tilde{L}$ to be the figure-eight knot of Figure $4($ a), let $N(\tilde{K})$ be trivial in $S^{3}$, and take $A=\left(\begin{array}{cc}\alpha & 2 \\ \beta & \delta\end{array}\right)$ (respectively, $\left(\begin{array}{cc}2 \alpha & 1 \\ \beta & \delta\end{array}\right)$ ). The manifold obtained by $2 / \delta$-surgery ( $\delta$ odd) (respectively, $1 / \delta$-surgery) on $\tilde{L}$ is a two-fold branched covering space of $S^{3}$ in the way depicted in Figure 15 (Figure 16, respectively).

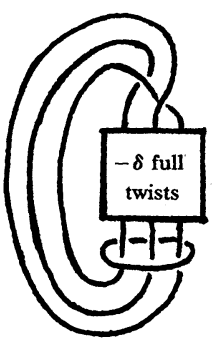

FIGURE 15

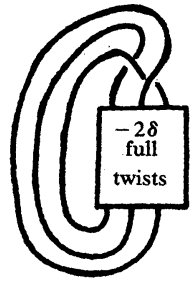

FIGURE 16 
On the other hand, since the figure-eight knot is strongly invertible in two ways (Figures 4(b) and (c)), the manifold obtained by $\gamma / \delta$-surgery on the figure-eight is a two-fold branched covering space of $S^{3}$ in two ways (Figure 17).
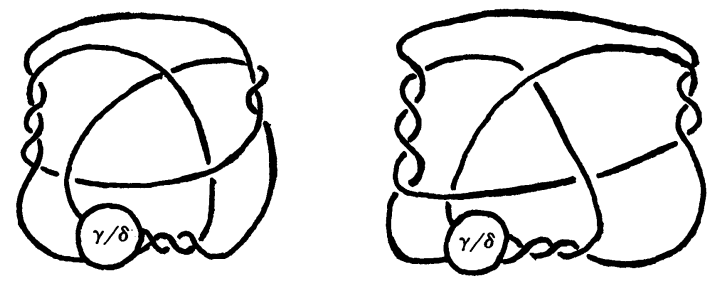

FigURE 17

REMARKS. (1) For $\gamma / \delta=m$, the knots in Figure 17 are 3-bridge knots, and their Conway potential functions show that they are of different types, if $m \neq 0$ (S. A. Bleiler has also noted this [B1]). By the method used in [BGM], it follows that the manifold obtained by $m$-surgery $(m \neq 0)$ on the figure-eight knot has at least two different Heegaard splittings. Since some of these manifolds are hyperbolic [Th], this answers the question of W. Jaco: Can there exist inequivalent, minimal, Heegaard splittings of a hyperbolic manifold?

Jozef Przytycki has pointed out that since the knots of Figure 17 are 3-braid knots, there exist infinitely many 3-manifolds with two different open-book decompositions of genus 1; the simplest such example is obtained by 5 -surgery on the figure-eight knot.

(2) For $\gamma / \delta=-1$, the knots of Figure 17 take the form of those in Figure 18. (Note that the knot of Figure 16 (with $\delta=-1$ ) coincides with that of Figure 18(a).) The knot of Figure 18(b) is the left-handed $(3,7)$-torus knot. The manifold $M$ is the Seifert fibered manifold (O o; $0 \mid-1 ; 2,3,7)$ (see [M]). We also get $M$ by a +1 -surgery on the left-handed trefoil, as Figure 14(b) shows (cf. [G], [BGM], and [Ta]).

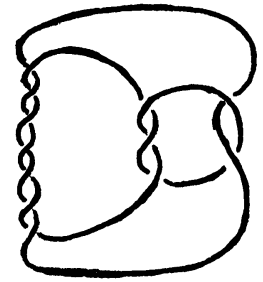

(a)

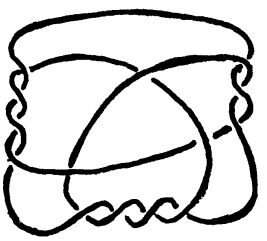

(b)

FIGURE 18 
(3) Note that the manifold obtained by $\gamma / \delta$-surgery on the figure-eight knot is the same as that obtained by $(-\gamma / \delta)$-surgery.

(4) As observed in [Th], the manifolds obtained by $\pm 1-, \pm 2-$ and \pm 3 -surgery on the figure-eight knot are Seifert fibered manifolds. The one obtained by \pm 4 -surgery is a graph manifold. We recognize these manifolds in Figure 19 [M].

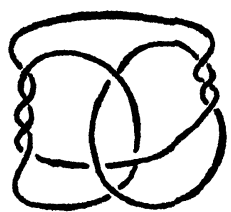

$m=-2$

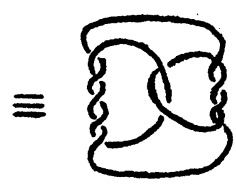

$(\mathrm{Oo} ; 0$ 이 $-1 ; 2 / 1 ; 4 / 1 ; 5 / 1)$

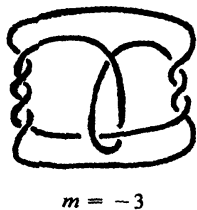

$(00 ; 0 \mid-1 ; 3 ; 3 ; 4)$
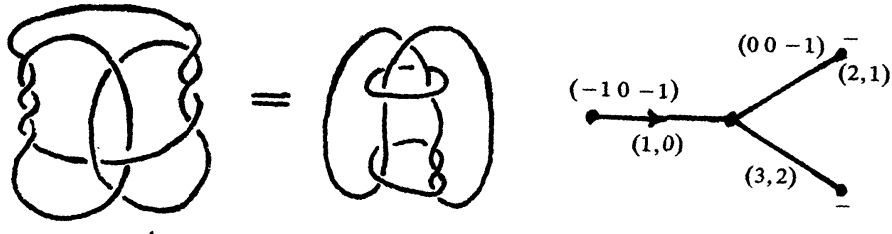

FIGURE 19

(5) For $\gamma / \delta=1 / \delta(\delta \neq-1)$, the manifold is the two-fold branched covering space of the knots in Figure 20, which are probably of different knot types. Because they have three local maxima, the corresponding manifold would have three different Heegaard splittings.
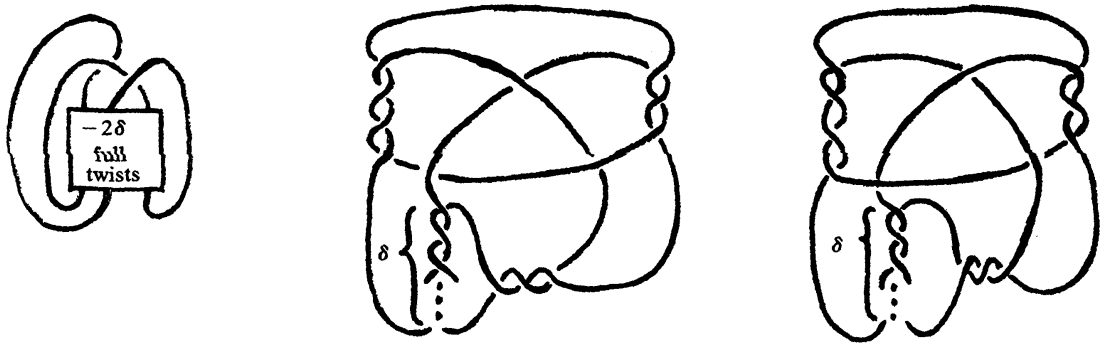

FIGURE 20 
REMARK. That the knots in Figure 20 for Example c(5) are, in fact, of different type for $\delta \neq 0$ was kindly pointed out to us by M. Sakuma (see [FS]). The two authors of [FS] obtained this result independently. As noted on page 191 of [FS], part of the result was known to M. Takahashi $\left[\mathbf{T a}_{\mathbf{1}}\right]$.

(d) Take $\tilde{L}$ to be the trefoil knot of Figure 3, take $N(\tilde{L})$ to be the knot $\tilde{K}$ of Figure 6 , and set $A=\left(\begin{array}{ll}\alpha & 2 \\ \beta & \delta\end{array}\right)$ (respectively, $\left.A=\left(\begin{array}{ll}0 & 1 \\ 1 & \delta\end{array}\right)\right)$. The manifold $M$ of Theorem 2.2 is a two-fold branched covering space of $S^{3}$ (Figures 21 and 22). Since both $\tilde{L}$ and $N(\tilde{L})$ are strongly invertible, the manifold $M$ is a two-fold branched covering space of $S^{3}$ in another form (Figure 23).

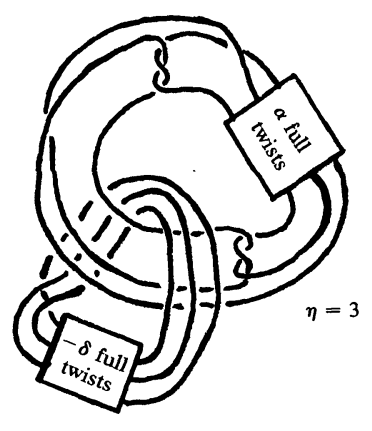

FIGURE 21

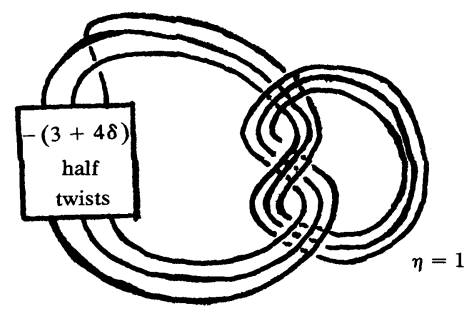

FIGURE 22

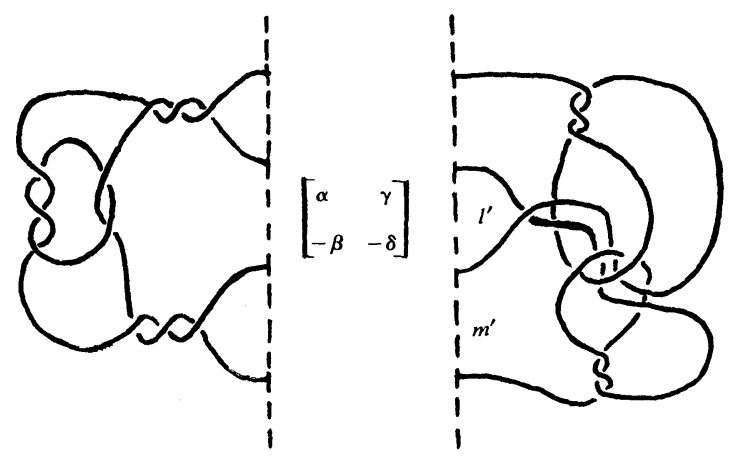

FigURE 23

REMARK. For $\eta=1$, the manifold $M$ corresponding to $A=\left(\begin{array}{ll}0 & 1 \\ 1 & \delta\end{array}\right)$ (Figure 22) is the result of 3-surgery on a double of the trefoil [BoGM].

(e) Take $\tilde{L}$ to be the knot $\tilde{K}$ of Figure 8 , take $N(\tilde{L})$ to be the trivial knot in $S^{3}$, and take $A=\left(\begin{array}{cc}\alpha & 2 \\ \beta & \delta\end{array}\right),\left(\begin{array}{cc}2 \alpha & 1 \\ \beta & \delta\end{array}\right)$, or $\left(\begin{array}{cc}\alpha & 1 \\ \beta & 2 \delta\end{array}\right)$. The corresponding manifolds $M_{A}$ are two-fold branched covering spaces of $S^{3}$, and the branch sets are depicted in Figure 24. 


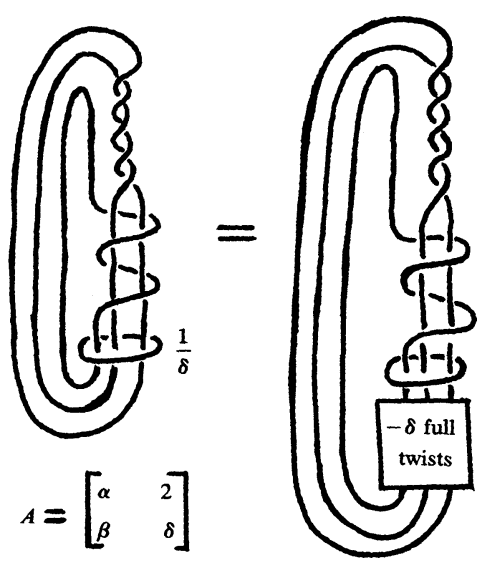

(a)

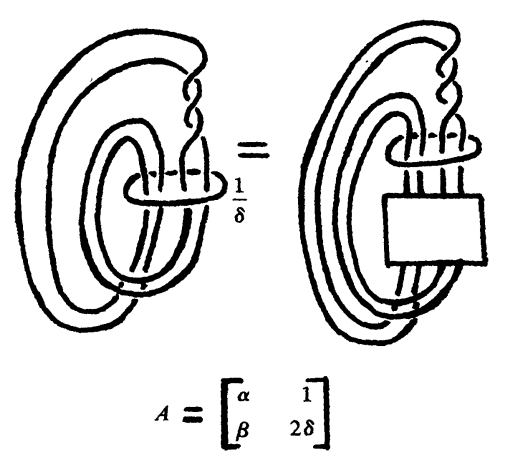

(c)

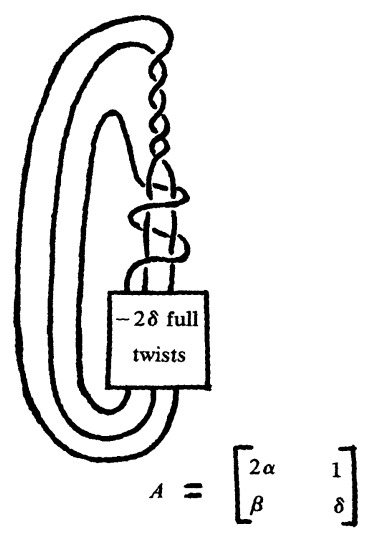

(b)

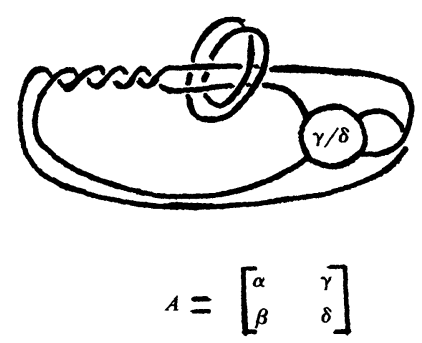

(d)

FigURE 24

REMARKS.

(1) For $A=\left(\begin{array}{ll}\alpha & 1 \\ \beta & 2\end{array}\right)$, the manifold $M_{A}$ is the result of 0 -surgery on the trefoil, and so $M_{A}$ is a torus bundle over $S^{1}$ with periodic monodromy; in Seifert's notation, $M_{A}=(\mathrm{Oo} ; 0 \mid-1 ; 2,3,6)(\mathrm{cf},[\mathbf{M}])$. The branch set is shown in Figure 25(a). The branch set of Figure 25(b) comes from Figure 14. That these two branch sets (Figure 25) are equal was pointed out to us by Sakuma (see his paper [Sa]).

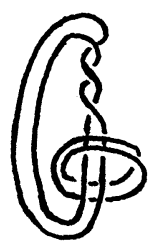

(a)

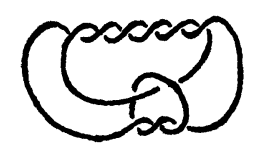

(b) 
(2) For $A=\left(\begin{array}{ll}\alpha & 1 \\ \beta & 0\end{array}\right)$, the manifold $M_{A}$ is the result of 0 -surgery on the figure-eight knot. The branch set is shown in Figure 26 in two ways (corresponding to the fact that $M_{A}$ is symmetric).
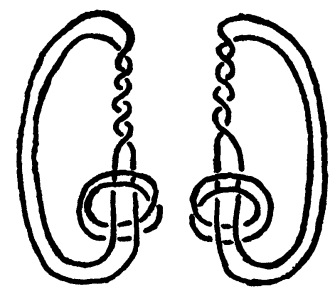

FIGURE 26

(f) Take $\tilde{L}$ to be the knot $\tilde{K}$ of Figure 8, let $N(\tilde{L})$ be the trefoil knot, and let $A$ be $\left(\begin{array}{ll}\alpha & 2 \\ \beta & \delta\end{array}\right)$ or $\left(\begin{array}{cc}\alpha & 1 \\ \beta & 2 \delta\end{array}\right)$. Then $M_{A}$ is a two-fold branched covering space of $S^{3}$ and, in each case, is the result of 0-surgery on a double of the trefoil (cf. [BoGM]). The branch sets are shown in Figure 27(a) and (b).

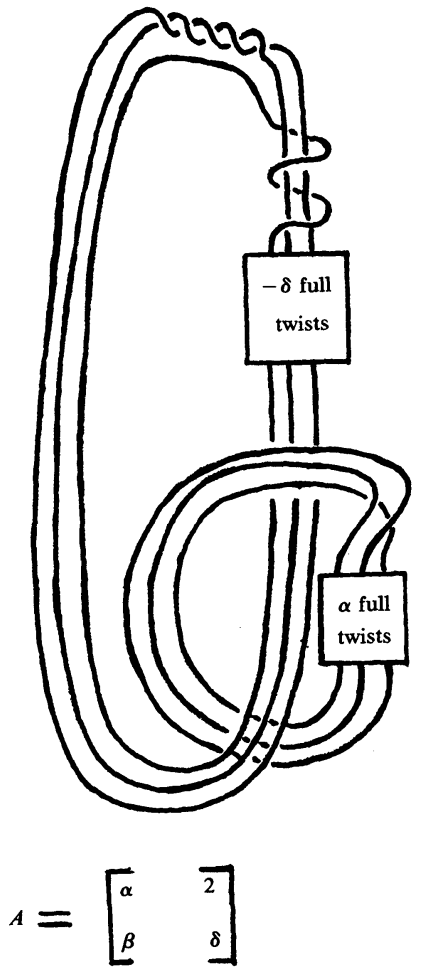

(a)

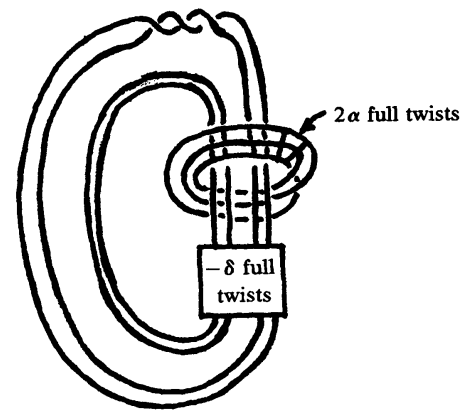

$$
A=\left[\begin{array}{rr}
\alpha & 1 \\
\beta & 2 \delta
\end{array}\right]
$$

(b)

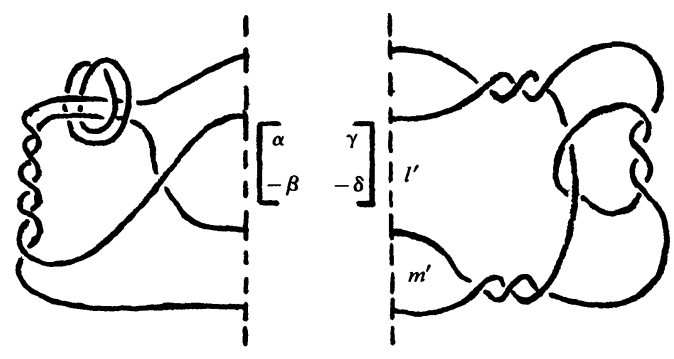

(c) 
Since both $\tilde{L}$ and $N(\tilde{L})$ are strongly invertible we get another two-fold branched covering space of $S^{3}$, whose branch set is shown in Figure 27(c).

(g) The link $\tilde{K}_{1} \cup \tilde{K}_{2}\left(=\tilde{L} \subset S^{3}\right)$ of Figure 28 is interchangeable in two ways, strongly invertible, and periodic. Figures 28(a) and (c) show (respectively) the orbit spaces $V$ and $U$ of $E\left(\tilde{K}_{1} \cup \tilde{K}_{2}\right)$ under involutions (rotations) of $S^{3}$ with fixed-point sets $A$ and $C$; the fixed-point set $A$ is perpendicular to the plane of projection of $\tilde{K}_{1} \cup \tilde{K}_{2}$. Figure $28(\mathrm{~d})$ and the top portion of (b) show the images of $\tilde{K}_{1} \cup \tilde{K}_{2}$ under covering maps induced by involutions (rotations) of $S^{3}$ with fixed-point set $F$ (a circle in a plane perpendicular to the plane of projection of $\tilde{K}_{1} \cup \tilde{K}_{2}$ and indicated by two crosses) and $B$, respectively. The bottom portion of Figure 28(b) shows the orbit space, $S^{2} \times I$, of $E\left(\tilde{K}_{1} \cup \tilde{K}_{2}\right)$ under an involution of $S^{3}$ with fixed-point set $B$. The fixed point sets $A, B$, and $C$ are mutually
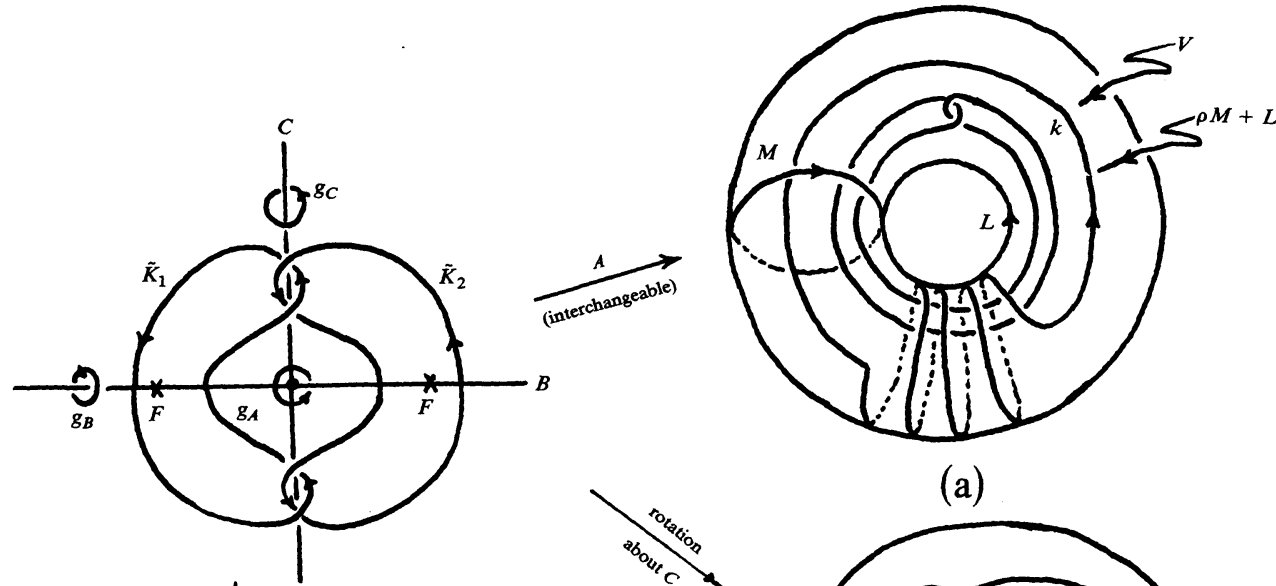

(a)
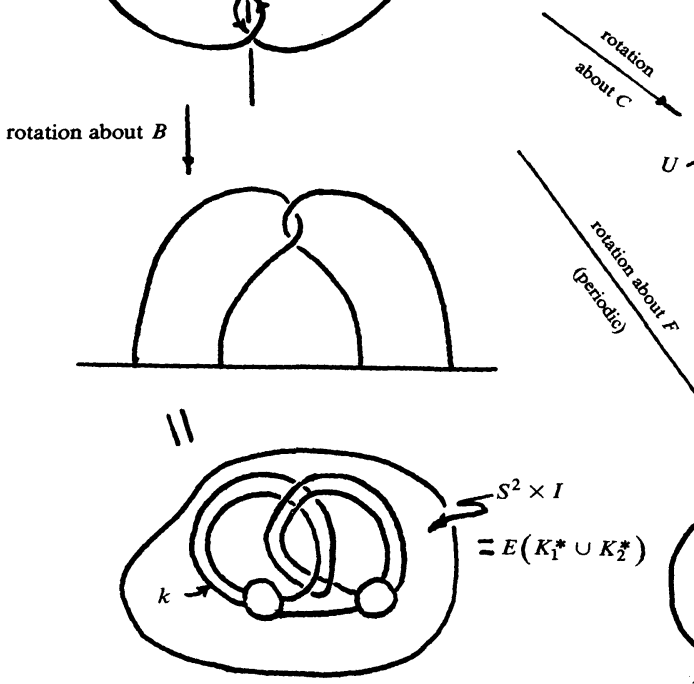

(b)

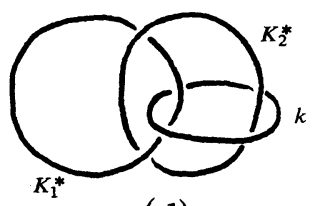

(c)

(d) 
perpendicular in $R^{3} \cup\{\infty\}\left(=S^{3}\right)$, and in all four cases $k$ denotes the image of the fixed-point set under the particular covering projection. We shall denote the restrictions of the four involutions of $S^{3}$ to $E\left(\tilde{K}_{1} \cup \tilde{K}_{2}\right)$ by $g_{A}, g_{B}, g_{C}$, and $g_{F}$; note that $\operatorname{Fix}\left(g_{B}\right)$ (the fixed-point set of $\left.g_{B}\right)$ is a collection of four arcs properly imbedded in $E\left(\tilde{K}_{1} \cup \tilde{K}_{2}\right)$. Finally, we can choose tubular neighborhoods of $\tilde{K}_{1}$ and $\tilde{K}_{2}$ so that $E\left(\tilde{K}_{1} \cup \tilde{K}_{2}\right)$ remains invariant under all four rotations of $S^{3}$.

Set $\tilde{V}=E\left(\tilde{K}_{1} \cup \tilde{K}_{2}\right)$, and let $T_{i}$ be the boundary component of $\tilde{V}$ corresponding to $\tilde{K}_{i}(i=1,2)$; note that $g_{A}\left(T_{i}\right)=T_{j}(i \neq j)$. Let $K$ be an oriented knot in $S^{3}$, set $N\left(\tilde{K}_{1}\right)=N\left(\tilde{K}_{2}\right)=K$, and let $\left(\tilde{m}^{\prime}, \tilde{l}^{\prime}\right)$ be the coordinate pair - in this case, a meridian-longitude pair - for $K$ on $\partial E(K)$. Finally, let $E_{1}(K)$ and $E_{2}(K)$ be copies of $E(K)$ with corresponding copies $\left(\tilde{m}_{1}^{\prime}, \tilde{l}_{1}^{\prime}\right)$ and $\left(\tilde{m}_{2}^{\prime}, \tilde{l}_{2}^{\prime}\right)$ of $\left(\tilde{m}^{\prime}, \tilde{l}^{\prime}\right)$, and let $\rho$ denote a fixed integer.

(1) By Theorem 2.2(vi) with pasting matrix $\left(\begin{array}{ll}0 & 1 \\ 1 & \rho\end{array}\right)$, the involution $g_{A}$ on $\tilde{V}$ extends to an involution $h_{A}$ on a two-fold branched covering space $\tilde{D}$ of $S^{3}$; let $p: \tilde{D} \rightarrow S^{3}$ denote the covering map that $h_{A}$ induces. The branch set $p A$ is a doubled knot - the double of $K$ with twist $\rho$. Set $D(K, \rho)=p A$, and write $\tilde{D}=E_{1}(K) \cup_{T_{1}} \tilde{V} \cup_{T_{2}} E_{2}(K)$.

REMARK. Note that (for fixed $\rho \neq 0$ ) the knot type of $D(K, \rho)$ depends on the isotopy type of $K$ and that our class of doubled knots contains all doubled knots up to knot type. We only need the stricter doubled-knot notation, $D(K ; \rho, \eta)$, for the statement of Corollary 3.10.

(2) Again, by Theorem 2.2(vi) with pasting matrix $\left(\begin{array}{ll}0 & 1 \\ 1 & \rho\end{array}\right)$, the involution $g_{C}$ on $\tilde{V}$ extends to an involution on a two-fold branched covering space $M$ of $S^{3}$. The manifold $M$ is the two-fold branched covering space of a $(4 \rho+1,4)$-cable about $K$ (Figure 28(c)). We thank W. B. R. Lickorish for pointing out that in this case, it is necessary to change the orientation of $\tilde{K}_{1}\left(\right.$ or $\left.\tilde{K}_{2}\right)$ in Figure 28 so that the oriented link $\tilde{K}_{1} \cup \tilde{K}_{2}$ is interchangeable with respect to $g_{C}$. Thus, the coordinate pairs for $\tilde{K}_{1}$ (or $\tilde{K}_{2}$ ) (see Proposition 1.1) in (1) and (2) differ in the $\tilde{l}$-coordinate by $\pm 4 \tilde{m}$, and so $M \cong \tilde{D}$ if and only if $M \cong S^{3} \cong \tilde{D}$, which is clear from $g(4)$ (below).

(3) If $K$ is strongly invertible, then the involution $g_{B}$ extends to an involution $h_{B}$ on $\tilde{D}$ (by Theorem 2.2(v)) that induces a two-fold branched covering map $p^{\prime}: \tilde{D} \rightarrow S^{3}$. The Fix $\left(h_{B}\right)$ is a simple closed curve whose intersection with $\tilde{V}$ is $\operatorname{Fix}\left(g_{B}\right)$; abusing notation, we set $B=\operatorname{Fix}\left(h_{B}\right)$. Hence, the branch set $p^{\prime}(B)$ is a knot in $S^{3}$ (Figure 28(b)); set $D^{\prime}=p^{\prime} B$. By Proposition 3.8, the knot $D^{\prime}$ is not a doubled knot, when $K$ is prime.

(4) If $K$ is invertible, then $g_{C}$ extends to an involution $h_{C}$ on $\tilde{D}$. To obtain $\tilde{D} / h_{C}$, we attach the boundary of the solid torus $U$ (Figure 28(c)), 
to $\partial E(K)$ by a homeomorphism $\psi: \partial U \rightarrow \partial E(K)$ such that

$$
(\psi M, \psi L) \sim\left(\tilde{m}^{\prime}, \tilde{l}^{\prime}\right)\left(\begin{array}{cc}
1+4 \rho & -\rho \\
-4 & 1
\end{array}\right) .
$$

If $\rho \neq 0$, then $\tilde{D} / h_{C}$ is clearly not $S^{3}$. If $\rho=0$ and if we attach a 2-handle to $E(K)$ along the curve $\tilde{m}^{\prime}-4 \tilde{l}^{\prime}$ on $\partial E(K)$, then there is (at the present time) a possibility for obtaining a homotopy 3-cell ( $K \mathrm{might}$ not have Property P).

(5) Suppose that $g_{F}$ extends to an involution $h_{F}$ on $\tilde{D}$ with $\operatorname{Fix}\left(h_{F}\right)$ $=F$ (Figure 28$)$. To see this involution, we consider the link $\tilde{K}_{1} \cup \tilde{K}_{2}$ of Figure 28 as a $(2,4)$-torus link $\tilde{L}$ on a standardly imbedded torus $T$ in $S^{3}$; we have $E(\tilde{L})=\tilde{V}$. The $\operatorname{Fix}\left(h_{F}\right)$ is now the core of the solid torus bounded by $T$ such that $\left|\operatorname{Lk}\left(\tilde{K}_{i}, F\right)\right|=1(i=1,2)$. The orbit space $\tilde{V} / g_{F}$ ( $\left.\cong S^{1} \times S^{1} \times I\right)$ is the exterior of the Hopf link $K_{1}^{*} \cup K_{2}^{*}$ of Figure 28(d). Moreover, if $K$ is knotted, then each of $E_{1}(K) / h_{F}$ and $E_{2}(K) / h_{F}$ is a knot manifold (not a solid torus), if $\tilde{D} / h_{F} \cong S^{3}$, because the two-fold unbranched covering space of a solid torus is a solid torus. But then $E_{2}(K) / h_{F}$ would belong to the solid torus $S^{3}-\operatorname{Int}\left(E_{1}(K) / h_{F}\right)$ in a nontrivial way (not in a 3-cell in the solid torus). This is impossible [BM]; hence, if $K$ is nontrivial and if $g_{F}$ extends to $\tilde{D}$, then $\tilde{D} / h_{F} \neq S^{3}$.

(6) Since $\tilde{K}_{1} \cup \tilde{K}_{2}$ is a $(2,4)$-torus link, there is one final obvious involution $g_{f}$ of $E\left(\tilde{K}_{1} \cup \tilde{K}_{2}\right)$. This is a free involution that interchanges the boundary components of $E\left(\tilde{K}_{1} \cup \tilde{K}_{2}\right)$. We will show that later (Theorem 3.6) that the five involutions $g_{A}, g_{B}, g_{C}, g_{F}$, and $g_{f}$ on $\tilde{V}$ are the only possible ones, up to strong equivalence (defined in the next section). One can easily see, by considering orbit spaces and fixed-point sets, that no two of these involutions are strongly equivalent.

REMARK. Example (g) is important, because it forms the core of the next section.

In the statement of Theorem 2.3, we shall use the concept of surgery instruction $\chi$. Given a link in $S^{3}$, the function $\chi$ associates to each component of the link a rational number and determines, in the usual way, a manifold obtained by Dehn surgery on the link.

THEOREM 2.3. Let $\tilde{L}$ be a 2-symmetric link in $\tilde{M}$ such that the link part of $L$ admits a surgery instruction $\chi$ that produces $S^{3}$. Assume also that

(i) if $\tilde{K}_{1} \cup \tilde{K}_{2}$ is an interchangeable sublink of $\tilde{L}$, then both $N\left(\tilde{K}_{1}\right)$ and $N\left(\tilde{K}_{2}\right)$ are trivial in $S^{3}$, the matrix $A=\left(\begin{array}{cc}\alpha & \gamma \\ \beta & \delta\end{array}\right)$, and $\chi\left(p \tilde{K}_{1}\right)=\gamma / \delta$; 
(ii) if $\tilde{K}$ and $N(\tilde{K})$ are both periodic, then the orbit space of $E(N(\tilde{K}))$ is a solid torus, the matrix $A=\left(\begin{array}{cc}\alpha & 2 \gamma \\ \beta & \delta\end{array}\right)$, and $\chi(p \tilde{K})=\gamma / \delta$;

(iii) if $\tilde{K}$ is periodic and $N(\tilde{K})$ is invariant, then the orbit space of $E(N(\tilde{K}))$ is a solid torus, $A=\left(\begin{array}{c}2 \alpha \\ \beta\end{array}\right)$, and $\chi(p \tilde{K})=\gamma / 2 \delta$;

(iv) if $\tilde{K}$ is invariant and $N(\tilde{K})$ is periodic, then the orbit space of $E(N(\tilde{K}))$ is a solid torus, $A=\left(\begin{array}{cc}\alpha & \gamma \\ \beta & 2 \delta\end{array}\right)$, and $\chi(p \tilde{K})=\gamma / \delta$;

(v) if $\tilde{K}$ and $N(\tilde{K})$ are both invariant, then the orbit space of $E(N(\tilde{K}))$ is a solid torus, $A=\left(\begin{array}{c}\alpha \gamma \\ 2 \beta \delta\end{array}\right)$, and $\chi(p \tilde{K})=2 \gamma / \delta$; and

(vi) if $K$ is strongly invertible, then so is $N(\tilde{K})$. Then, the resulting oriented, closed 3-manifold $M$ admits an involution whose orbit space is $S^{3} . \square$

3. Double covers of doubled knots. So far, we have constructed a variety of 3-manifolds with nonunique representations as two-fold branched covering spaces of $S^{3}$. The uniqueness question, however, is also of interest. In fact, in [BGM; $§ 1$, p. 98], the authors ask for a cataloguing of those knots (and links) in $S^{3}$ whose two-fold branched covering spaces characterize their type, and some basic cases are already known to be on the list. Results of F. Waldhausen $\left[\mathbf{W}_{2}\right]$, J. L. Tollefson [Tol $\left.{ }_{1}\right]$, and C. Hodgson [Ho] show that $S^{3}$, and $S^{2} \times S^{1}$, and all lens spaces have unique representations as two-fold branched covering spaces of $S^{3}$, and J. H. Rubinstein [Ru] has shown that certain Seifert fibered 3-manifolds also have this property. In this section, we extend this list to include the two-fold branched covering spaces of all the doubles (with nontrivial twisting) of any prime knot that is not strongly invertible. (Note that this is a larger class of knots than that covered in the theorem of our abstract [MW]; we do not require that the exterior of the prime knot that we are doubling contain no essential annuli.)

Much of the notation that we shall use comes from Example $(\mathrm{g})$ of the previous section and will not generally by referenced or explained in the present section. We begin with several lemmas leading to our Theorems 3.6 and 3.7.

Autohomeomorphism $h$ and $g$ of a manifold $M$ are strongly equivalent, if there exists a homeomorphism $f: M \rightarrow M$ such that $f$ is isotopic to the identity and such that $h=f g f^{-1}$. To prove our main result (Theorem 3.7) of this section, we need to classify the strong equivalence classes of involutions on $\tilde{V}$. For this we need several lemmas. Note first that $\tilde{V}$ is a Seifert-fibered solid torus of type $(2,1)$ [Ja; p. 84] with a fibered neighborhood of a regular fiber removed $\left(\tilde{V}\right.$ is a bundle over $S^{1}$ with a disk-with-two-holes as the fiber). Hence, $\tilde{V}$ has one exceptional fiber $\left(=\operatorname{Fix}\left(g_{F}\right)=F\right)$, and the orbit manifold $V_{0}$ of $\tilde{V}$ is an annulus. 
LEMMA 3.1. The only closed incompressible surfaces in the two-component link exterior $\tilde{V}$ are boundary-parallel tori.

Proof The space $\tilde{V}$ is the exterior of a $(2,4)$-torus link (the link $\tilde{K}_{1} \cup \tilde{K}_{2}$ of Figure 28 ), which is a 2-bridge link, and it is well known that the exterior of a 2-bridge link contains no closed nonperipheral incompressible surfaces (see, for example, [GL; Corollary 1.2].

REMARK. Note that $\tilde{V}$ is the exterior of a well-defined infinite class of links [BuM], but we shall be interested only in $\tilde{K}_{1} \cup \tilde{K}_{2}$.

A surface in a 3-manifold is essential, if it is properly imbedded, incompressible, boundary incompressible, and not boundary parallel. A surface in a Seifert fibered manifold is fiber complete, if the surface is a union of (Seifert) fibers; this is a variation of the term "saturated" [Ja; p. 87]. By a "surface," we shall always mean a "connected surface."

LEMMA 3.2. The manifold $\tilde{V}$ contains exactly three distinct ambient-isotopy classes of essential annuli, each containing a fiber-complete representative.

Proof. To obtain representatives of the three classes, we lift three, suitable, properly imbedded arcs (missing the exceptional point) in the orbit manifold $V_{0}$ to $\tilde{V}$. The boundary of one of these annuli is in $T_{1}$, the boundary of another is in $T_{2}$, and the third annulus has a boundary component in each of $T_{1}$ and $T_{2}$. Obviously, these are the only fiber-complete essential annuli in $\tilde{V}$ (up to isotopy). But, in $\tilde{V}$, any essential annulus is isotopic to one that is either fiber complete or transverse to all the (Seifert) fibers [ $\left.\mathbf{W a}_{\mathbf{1}}\right]$. The restriction of the projection map $\tilde{V} \rightarrow V_{0}$ to an annulus transverse to all the fibers would, however, yield a covering with exactly one branch point of an annulus by an annulus. Since an Eulercharacteristic argument shows that this is impossible, our conclusion follows.

REMARK. Bleiler has also observed Lemmas 3.1 and 3.2 [Bl].

LEMMA 3.3. Let $Q$ be a (fixed) annulus from the unique isotopy class of essential annuli in $\tilde{V}$ (Lemma 3.2) with one boundary component in each component of $\partial \tilde{V}$, and let $h$ be a PL involution on $\tilde{V}$. Then there exists a homeomorphism, $\sigma: \tilde{V} \rightarrow \tilde{V}$, such that $\sigma$ is isotopic to the identity and $\sigma h \sigma^{-1}(Q)=Q$. 
Proof. By [ $\mathbf{T o l}_{2}$ ], we can assume that $h$ preserves the Seifert-fiber structure on $\tilde{V}$. Hence, $h$ induces an involution $h_{0}$ on the orbit manifold $V_{0}$; if $e: \tilde{V} \rightarrow V_{0}$ is the projection map, then $e h=h_{0} e$, and the exceptional point $v_{0}$ in $V_{0}$ (corresponding to the exceptional fiber in $\tilde{V}$ ) is a fixed point of $h_{0}$.

Now considering $V_{0}$ as $S^{1} \times I$, we can find a product fibering of $V_{0}$ and an involution $g$ of $S^{1}$ such that, relative to this fibering, $h_{0}(x, t)=$ $(g(x), k(t))$, in which $k(t)=t$ or $1-t$, for $(x, t) \in S^{1} \times I$; this is just a two-dimensional analogue of Theorem 1.3 of [Ki]. Then there exists a unique point $x_{0} \in S^{1}$ such that $\left\{x_{0}\right\} \times I$ contains the exceptional point $v_{0}$, and so $h_{0}\left(\left\{x_{0}\right\} \times I\right)=\left\{x_{0}\right\} \times I$. Splitting $V_{0}$ along $\left\{x_{0}\right\} \times I$, we obtain a disk $D_{0}$, which must contain a fixed point $\left(x_{1}, t_{1}\right)$ of $h_{0}$. Assuming, as we can, that $D_{0}$ is the complement in $V_{0}$ of a small $h_{0}$-invariant neighborhood of $\left\{x_{0}\right\} \times I$, it follows that $h_{0}\left(\left\{x_{1}\right\} \times I\right)=$ $\left\{x_{1}\right\} \times I \neq\left\{x_{0}\right\} \times I$.

Let $Q^{\prime}=e^{-1}\left(\left\{x_{1}\right\} \times I\right)$. Then $Q^{\prime}$ is isotopic to $Q$ in $\tilde{V}$ and $h\left(Q^{\prime}\right)=$ $Q^{\prime}$. To complete the proof, let $\sigma$ be any homeomorphism on $\tilde{V}$ such that $\sigma$ is isotopic to the identity and $\sigma Q^{\prime}=Q$. Then $\sigma h \sigma^{-1}(Q)=Q$.

LEMma 3.4. Let $W$ be a Seifert-fibered solid torus and let $h$ be a PL involution on $W$ preserving the fibration. Then there exists a meridional disk $D$ of $W$ that is transverse to the fibration and to the $\operatorname{Fix}(h)$, and for which either $h(D) \cap D=\varnothing \operatorname{or} h(D)=D$.

Proof. The proof is the same as that of Lemma 3 in [KT $\mathbf{T}_{\mathbf{2}}$, p. 267] except that admissible disks (the collection $\Sigma$ in the proof of $\left[\mathbf{K T}_{\mathbf{2}}\right.$; Lemma 3]) are transverse to the fibration, and the $h$-general position isotopies and the isotopies of $\alpha$-operations and $\beta$-operations [KT, $\mathrm{pp}$. $223,224,226$ ] are to be taken as fiber preserving (cf. proof of Corollary 4.3 of $\left[\mathrm{Tol}_{3}\right.$; p. 334]). (Note that $\Sigma \neq \varnothing$, because we can easily find a meridional disk transverse to the fibering and then put it into $h$-general position by a fiber-preserving isotopy.)

REMARKS. The conclusion of Lemma 3.4 also holds when $W$ is a solid Klein bottle, but Lemma 3.4 cannot be extended to any other Seifert fibered manifold, because a solid torus and a solid Klein bottle are the only Seifert fibered manifolds with a compressible boundary component.

LEMMA 3.5. Let $\left\{A_{1}, \ldots, A_{n}\right\}$ be a nonempty collection of disjoint fiber-complete annuli on the boundary of a Seifert-fibered solid torus $W$ of type $(p, q)((p, q)=1$ and $0<q \leq p / 2)$, and let $h_{1}$ and $h_{2}$ be PL 
involutions on $W$ such that $h_{1}\left|A_{i}=h_{2}\right| A_{i}$ and $h_{j}\left(A_{i}\right) \in\left\{A_{1}, \ldots, A_{n}\right\}(i=$ $1, \ldots, n ; j=1,2)$. Then both $h_{1}$ and $h_{2}$ are OP (orientation preserving), and there exists a homeomorphism $f: W \rightarrow W$ isotopic to the identity $\operatorname{rel}\left(A_{1} \cup \cdots \cup A_{n}\right)$ for which $h_{2}=f h_{1} f^{-1}$.

Proof. Let $\tau$ be a boundary component of $A_{1}$, and let $(\mu, \lambda)$ be a nonoriented meridian-longitude pair for $W$ such that $\mu$ meets each (Seifert) fiber of $\partial W$ exactly $p$ times and $\lambda$ meets each such fiber exactly $q$ times. Orient $\lambda$. Then, for suitable orientations of $\mu$ and $\tau$, we have $\tau \sim q \mu+p \lambda$ on $\partial W$. If $h_{j}(j=1,2)$ were OR (orientation reversing), then either $h_{j}(\tau) \sim q \mu-p \lambda$ or $h_{j}(\tau) \sim-q \mu+p \lambda$ (on $\partial W$ ). But $h_{j}(\tau) \sim$ $\pm(q \mu+p \lambda)$ (because $\left.h_{j}\left(A_{1}\right) \in\left\{A_{1}, \ldots, A_{n}\right\}\right)$, and neither $q \mu+p \lambda$ nor $-(q \mu+p \lambda)$ is homologous to either $q \mu-p \lambda$ or $-q \mu+p \lambda$ (because $p, q \neq 0)$. Hence, both $h_{1}$ and $h_{2}$ are OP.

Choose a $(p, q)$-fibering on $\partial W$ that contains the curves in $\left\{\partial A_{1}, \ldots, \partial A_{n}\right\}$ as (regular) fibers and for which $h_{1} \mid \partial W$ is fiber preserving. To do this, cut $\partial W$ along each of the curves in $\left\{\partial A_{1}, \ldots, \partial A_{n}\right\}$. The closure of each component is an annulus and there are $2 n$ of them; let $A_{0}$ be one of them. Now either $h_{1}\left(A_{0}\right) \cap A_{0}=\varnothing$ or $h_{1}\left(A_{0}\right)=A_{0}$, and $\partial A_{0} \subset \partial A_{1} \cup \cdots \cup \partial A_{n}$. If $h_{1}\left(A_{0}\right) \cap A_{0}=\varnothing$, then any (Seifert) fibering of $A_{0}$ that includes $\partial A_{0}$ induces a fibering of $h_{1}\left(A_{0}\right)$ so that $h_{1}$ $\left(A_{0} \cup h_{1}\left(A_{0}\right)\right.$ ) is fiber preserving. If $h_{1}\left(A_{0}\right)=A_{0}$, then (as pointed out in the proof of Lemma 3.3), we can regard $h_{1} \mid A_{0}$ as a product map $\varphi \times \psi$ : $S^{1} \times I \rightarrow S^{1} \times I$ such that $\varphi^{2}=1$ and $\psi(t)=t$ or $1-t$, for each $t \in I$. Hence, we can take $\left\{S^{1} \times\{t\} \mid t \in I\right\}$ as an $h_{1}$-invariant fibering of $A_{0}$ when $h_{1}\left(A_{0}\right)=A_{0}$. Continuing through the remaining annuli of our cut-open version of $\mathrm{\partial} W$, just as for $A_{0}$, we can choose the fibering of $\mathrm{\partial} W$ to be $h_{1}$-invariant.

Now extend this fibering of $\partial W$ to a $(p, q)$-fibering $\mathscr{F}^{\prime}$ of $W$. By [Tol ${ }_{2}$; Lemma 6, p. 526], there exists a fiber structure $\mathscr{F}$ of $W$ such that $h_{1}$ preserves the fibers of $\mathscr{F}$ and such that $\mathscr{F}^{\prime}$ and $\mathscr{F}$ agree on $\partial W$.

By Lemma 3.4, there exists a meridional disk $D_{1}$ of $W$ that is transverse to both $\mathscr{F}$ and Fix $\left(h_{1}\right)$, and for which either $h_{1}\left(D_{1}\right) \cap D_{1}=\varnothing$ or $h_{1}\left(D_{1}\right)=D_{1}$. We now apply the proof of Lemma 3 of $\left[\mathbf{K T}_{2}\right]$ (this is just the equivariant-disk theorem referred to in the proof of Lemma 3.4 (above)) to find a second meridional disk $D_{2}$ of $W$ such that $D_{1} \cap$ $\left(A_{1} \cup \cdots \cup A_{n}\right)=D_{2} \cap\left(A_{1} \cup \cdots \cup A_{n}\right)$ (which is a collection of spanning arcs $\left\{a_{1}, \ldots, a_{p n}\right\}$ of $\left.A_{1}, \ldots, A_{n}\right)$, such that $D_{2}$ is transverse to $\operatorname{Fix}\left(h_{2}\right)$ and such that either $h_{2}\left(D_{2}\right) \cap D_{2}=\varnothing$ or $h_{2}\left(D_{2}\right)=D_{2}$. The set $\Sigma$ of admissible disks in the proof of Lemma 3 (of $\left[\mathbf{K T}_{\mathbf{2}}\right]$ ) is taken to be 
the set of all meridional disks of $W$ that intersect $A_{1} \cup \cdots \cup A_{n}$ in $a_{1} \cup \cdots \cup a_{p n}$ (only) and that are either $h_{2}$-invariant and in general position with respect to $\operatorname{Fix}\left(h_{2}\right)$ or are in $h_{2}$-general position; the proof of Lemma 3 (of $\left[\mathbf{K T}_{2}\right]$ ) then goes through with isotopies of $W$ that leave $A_{1} \cup \cdots \cup A_{n}$ pointwise fixed.

Since $\partial D_{j}$ meets each $A_{i}$ in $p(\geq 2)$ arcs, the collection of curves in $\left\{\partial D_{j}, h_{j}\left(\partial D_{j}\right), \partial A_{1}, \ldots, \partial A_{n}\right\}\left(=\Gamma_{j} ; j=1,2\right)$ induces an $h_{j}$-invariant cell decomposition $\mathscr{C}_{j}$ of $\partial W$; note that either $h_{1}\left(D_{1}\right) \cap D_{1}=\varnothing$ and $h_{2}\left(D_{2}\right)$ $\cap D_{2}=\varnothing$ or $h_{1}\left(D_{1}\right)=D_{1}$ and $h_{2}\left(D_{2}\right)=D_{2}$, because $h_{1}\left|A_{i}=h_{2}\right| A_{i}$ for each $i$. The 0 -cells of $\mathscr{C}_{j}$ are just the points in

$$
\left[\partial D_{j} \cup h_{j}\left(\partial D_{u}\right)\right] \cap\left[\partial A_{1} \cup \cdots \cup \partial A_{n}\right] .
$$

The 0-cells divide the curves of $\Gamma_{j}$ into arcs - the 1-cells of $\mathscr{C}_{j}$.

Since $\partial D_{1} \cap \partial D_{2} \cap\left(A_{1} \cup \cdots \cup A_{n}\right)$ is the collection $\left\{a_{1}, \ldots, a_{p n}\right\}$ of pn disjoint arcs (1-cells of both $\mathscr{C}_{1}$ and $\mathscr{C}_{2}$ ) and since $h_{1}\left|A_{i}=h_{2}\right| A_{i}$ $(i=1, \ldots, n)$, there is a homeomorphism $f_{1}: W \rightarrow W$ that is isotopic to the identity $\operatorname{rel}\left(A_{1} \cup \cdots \cup A_{n}\right)$ and that takes $D_{1}$ onto $D_{2}$ and $h_{1}\left(D_{1}\right)$ onto $h_{2}\left(D_{2}\right)$. Note that $f_{1}\left(\mathscr{C}_{1}\right)=\mathscr{C}_{2}$ and that $f_{1} h_{1} f_{1}^{-1}\left(\mathscr{C}_{2}\right)=\mathscr{C}_{2}$; we shall denote $f_{1} h_{1} f_{1}^{-1}$ by $h_{1}$ and say (following Hartley [Ha]) that we have varied $\left.h_{1}\right)$.

Obviously, $h_{1}$ and $h_{2}$ correspond on the 0-cells of $\mathscr{C}_{2}$. It is also obvious that, if $d$ is either a 1-cell or 2-cell of $\mathscr{C}_{2}$, then $h_{1}(d)=h_{2}(d)$; moreover, $h_{1}\left(D_{2}\right)=h_{2}\left(D_{2}\right)$. Consequently, by Hartley's trick (Lemma 2.1 and technique $A$ of [Ha] $)$, we can vary $h_{1} \mid\left(D_{2} \cup h_{2}\left(D_{2}\right) \cup \partial W\right)$ so that $h_{1}$ and $h_{2}$ correspond on $D_{2} \cup h_{2}\left(D_{2}\right) \cup \partial W$ (cf. [Ha, Proof of Lemma 2.3, p. 178]). Finally, if we split $W$ along the $\operatorname{disk}(\mathrm{s}), D_{2}$ and $h_{2}\left(D_{2}\right)$, and apply Hartley's trick to the resulting 3-cell(s), we can vary $h_{1}$ so that $h_{1}=h_{2}$ on $W$. Since all these variations of $h_{1}$ can be chosen to leave $A_{1} \cup \cdots \cup A_{n}$ pointwise fixed, the proof is complete.

Let $S$ be a properly imbedded, two-sided surface in a 3-manifold $M$ and let $\Sigma_{S}(M)$ denote $M$ split along $S$. There is a natural projection $\gamma$ : $\Sigma_{S}(M) \rightarrow M$, and $\gamma^{-1}(S)$ is the union of two copies of $S$ each of which is mapped by $\gamma$ homeomorphically onto $S$. An involution $g$ on $M$ with $g(S)=S$ lifts to an involution $\bar{g}$ of $\Sigma_{S}(M)$ such that $\gamma \bar{g}=g \gamma$, because $S$ is two-sided in $M$.

THEOREM 3.6. Let $k$ be any ( fixed) nonzero integer and let $V_{k}$ denote the Seifert fibered manifold $(\mathrm{O}, \mathrm{o}: 0 \mid 0 ; 2 k / 1)$ with fibered neighborhoods of two regular fibers removed. There exist exactly five (nontrivial) strong 
equivalence classes of PL involutions on $V_{k}$, and every PL involution on $V_{k}$ is orientation preserving. The fixed-point sets of these five classes are as follows: (1) $\varnothing$, for one class; (2) $S^{1}$, for three classes; and (3) four properly imbedded arcs, for one class.

Proof. The Seifert manifold $V_{k}$ is the exterior (in $\left.S^{3}\right)$ of $(2,4|k|)$-torus link (meeting each meridian (on some torus) twice and each longitude $4|k|$ times) and can also be obtained from $\left(S^{1} \times I\right) \times S^{1}$ by $(2 k / 1)$-surgery on a regular fiber in the (product) Seifert fibration. Note that the conclusions of Lemmas 3.1, 3.2 and 3.3 hold for each $V_{k}$ just as for $V_{-1}(=\tilde{V})$; the proofs are the same.

Let $g_{1}$ and $g_{2}$ be any two PL involutions on $V_{k}$, and let $A_{0}$ be an annulus from the unique isotopy class of essential annuli in $V_{k}$ with one boundary component in each boundary component of $V_{k}$ (Lemma 3.2). By Lemma 3.3, we can assume that $g_{i}\left(A_{0}\right)=A_{0}$ without changing the strong-equivalence class of $g_{i}(i=1,2)$. Now suppose that $g_{1} \mid A_{0}$ and $g_{2} \mid A_{0}$ are strongly equivalent. We will show that $g_{1}$ and $g_{2}$ are themselves strongly equivalent.

Because $g_{1} \mid A_{0}$ and $g_{2} \mid A_{0}$ are strongly equivalent and because $A_{0}$ is bicollared in $V_{k}$ by a product neighborhood meeting each component of $\partial V_{k}$ in an annulus, we can vary $g_{1}$ so that $g_{1}\left|A_{0}=g_{2}\right| A_{0}$; we assume that this has been done. The space $\Sigma_{A_{0}}\left(V_{k}\right)\left(=V_{k}\right.$ split along $\left.A_{0}\right)$ is a solid torus $W$; let $\gamma: W \rightarrow V_{k}$ denote the natural projection. Then $\gamma^{-1}\left(A_{0}\right)$ is a pair of disjoint annuli $\left\{A_{1}, A_{2}\right\}$ on the $\partial W$ each of whose median is a $(2|k|, 1)$-torus knot. If $\bar{g}_{1}$ and $\bar{g}_{2}$ are the lifts to $W$ of $g_{1}$ and $g_{2}$, then $\bar{g}_{j}\left(A_{i}\right) \in\left\{A_{1}, A_{2}\right\}(i=1,2 ; j=1,2)$. By the first paragraph in the proof of Lemma 3.5 , it follows that each of $\bar{g}_{1}$ and $\bar{g}_{2}$ is OP; consequently, each of $g_{1}$ and $g_{2}$ is also OP. Thus, by considering a small 3-cell $Q$ in $V_{k}$ that meets $A_{0}$ in a disk in $\partial Q$, one can easily show that both $g_{1}$ and $g_{2}$ preserve the sides of $A_{0}$ or both reverse them. But then, $\bar{g}_{1}\left(A_{i}\right)=\bar{g}_{2}\left(A_{i}\right)$ ( $i=1,2)$, and so $\bar{g}_{1}\left|A_{i}=\bar{g}_{2}\right| A_{i}$, because $g_{1}\left|A_{0}=g_{2}\right| A_{0}$. It follows immediately from Lemma 3.5 that $g_{1}$ and $g_{2}$ are strongly equivalent. Hence, if $g_{1} \mid A_{0}$ and $g_{2} \mid A_{0}$ are strongly equivalent, then so are $g_{1}$ and $g_{2}$.

As Kim noted in [Ki; p. 382] and as we noted earlier, any involution on $S^{1} \times I$ is strongly equivalent to one of the form $\varphi \times \psi$, with $\varphi^{2}=$ id and $\psi(t)=t$ or $1-t$, for each $t \in I$. Therefore, there are exactly five (nontrivial) strong-equivalence classes of involutions on $S^{1} \times I$ and, hence, at most five such classes of involutions on $V_{k}$.

As we have seen, however, there are at least five strong-equivalence classes of involutions (represented by $g_{A}, g_{B}, g_{C}, g_{F}$ and $g_{f}$ ) on $\tilde{V}$. As 
with the $(2,4)$-torus link $\tilde{K}_{1} \cup \tilde{K}_{2}$ (whose exterior is $\tilde{V}$ ), it is not difficult to see that a $\left(2,4|k|\right.$ )-torus link (whose exterior is $V_{k}$ ) is interchangeable in two ways, strongly invertible, periodic, and freely periodic (by an involution in $S^{3}$ ). Therefore, there are at least five strong-equivalence classes of involutions, each composed of OP involutions on $V_{k}$.

THEOREM 3.7. If $K$ is a prime knot that is not strongly invertible, then the two-fold branched cyclic covering space $\tilde{D}$ of a double $D(K, \rho)$ of $K$ characterizes the knot type of $D(K, \rho)$, if $\rho \neq 0$ (or if $K$ has property $P$ ).

Proof. We have $\tilde{D}=E_{1}(K) \cup_{T_{1}} \tilde{V} \cup_{T_{2}} E_{2}(K)$. Let $h_{A}$ be an OP involution such that $\operatorname{Fix}\left(h_{A}\right)=A$ (see Figure 28) and such that the induced covering map $p: \tilde{D} \rightarrow S^{3}$ maps $A$ onto $D(K, \rho)$. Let $h^{\prime}$ be a second OP involution on $\tilde{D}$ with $\mathrm{Fix}\left(h^{\prime}\right)$ a simple closed curve $A^{\prime}$. Moreover, suppose that $h^{\prime}$ induces a covering map $p^{\prime}: \tilde{D} \rightarrow S^{3}$ with $p^{\prime}\left(A^{\prime}\right)=D^{\prime}$. We shall show that $D(K, \rho)$ and $D^{\prime}$ belong to the same knot type, provided that the prime knot $K$ is not strongly invertible and that $\rho \neq 0$ (which is a technical condition to avoid the possibility that $K$ does not have property $P$ ).

Let $\Sigma$ denote a characteristic submanifold (called a characteristic Seifert pair in [Ja; p. 172]) for $\tilde{D}$. By [JS; Lemma V.3.3, p. 152], the inclusion map $i: \tilde{V} \rightarrow \tilde{D}$ is nondegenerate [JS; p. 55]. By [Ja; Theorem IX.17, p. 174], the inclusion map $i$ is homotopic to a map $g: \tilde{V} \rightarrow \tilde{D}$ such that $g(\tilde{V}) \subseteq \Sigma$, and so we can assume (after an ambient isotopy) that $\tilde{V} \subseteq \Sigma$ [JS; Squeezing Theorem, p. 139]. Since all incompressible tori in $\tilde{V}$ are boundary parallel (that is, $\tilde{V}$ is simple), we can assume that, in fact, $\tilde{V}$ is either a component of $\Sigma$ or contained in a nonsimple component $\sigma$ of $\Sigma$. Suppose that the latter holds. Then $\sigma=W_{1} \cup_{T_{1}} \tilde{V} \cup_{T_{2}} W_{2}$ for which at least one of $W_{1}$ and $W_{2}$ is not $\varnothing$.

By [JS; Corollary V.5.1, p. 164], $\tilde{D}$ contains a unique (up to ambient isotopy), mutually disjoint, minimal family of incompressible tori $T$ (a characteristic family) such that each component of $\Sigma_{T}(\tilde{D})$ is either a Seifert fibered manifold or a simple manifold. According to the splitting theorem [JS; p. 157], we can assume that $T \subseteq \partial \Sigma$; we can evidently also assume that $\partial \sigma \subseteq T$ [JS; Proposition V.4.4, p. 156].

Since $\sigma$ is not simple, it must be Seifert fibered [JS; Corollary V.5.1 p. 164]. We assume that $W_{1}(\subset \sigma)$ is not $\varnothing$. The torus $T_{1}$ is incompressible in $\sigma$ (since $K$ is nontrivial), and so $\pi_{1}(\sigma)$ contains an infinite cyclic normal subgroup [JS; Lemma II.4.2(i), p. 23] $N$. Evidently, $\pi_{1}(\sigma)$ is a nontrivial free product with amalgamation (on $\pi_{1}\left(T_{1}\right)$ ), since $K$ is a 
nontrivial knot and $W_{1} \not S^{1} \times S^{1} \times I$. Now suppose that there is no Seifert fibration of $\sigma$ in which $T_{1}$ is saturated (that is, fiber complete). Then $N \not \subset \pi_{1}\left(T_{1}\right)$ [Ja; VI.25, p. 102], and so $\pi_{1}\left(T_{1}\right)$ has index two in each of $\pi_{1}\left(W_{1}\right)$ and $\pi_{1}\left(\tilde{V} \cup W_{2}\right)$ [GH; Lemma 1, p. 305]. By the corollary of $\left[\mathbf{Z e}_{1}\right.$, p. 1116] (which is a corollary to the theorem of $\left[\mathbf{Z e}_{2}\right.$, p. 366]), it follows that $\pi_{1}\left(\tilde{V} \cup W_{2}\right) \approx Z \times Z$, which is not true, whether $W_{2}=\varnothing$ or not. Hence, there is a Seifert fibration of $\sigma$ in which $T_{1}$ is fiber complete, and so $W_{1}$ is Seifert fibered manifold.

By [Ja; Lemma IX.22, p. 188], $W_{1}$ is either a cable space, a torus-knot space, or a composing space (an $S^{1}$-bundle over a disk with $n$ holes). (It follows that $W_{2} \cong W_{1}$, because $E_{1}(K) \cong E_{2}(K)$, the family $T$ is minimal, and the fiber structure on $h_{A} W_{1}$ is compatible with that of $W_{1} \cup \tilde{V}$.) There is an essential fiber-complete annulus $A_{1}$ in $W_{1}$ with $\partial A_{1} \subset T_{1}$, and by the construction of $\tilde{D}, p\left(\partial A_{1}\right)$ is a pair of meridians of $V(=p \tilde{V})$ on $\partial V$ (cf. Figure 28(a)). But then $p A_{1}$ is an essential annulus in $E(K)$ whose boundary components are meridians of $K$, and so $W_{1}$ (as well as $W_{2}$ ) is a composing space; that is, $K$ is a composite knot, contrary to hypothesis. Therefore, $\sigma=\tilde{V}$; that is, $\tilde{V}$ is a component of $\Sigma$.

Since $\partial \sigma \subseteq T$, it follows that $\left\{T_{1}, T_{2}\right\} \subseteq T$. Now there exists a characteristic family of tori $T^{\prime}$ for $\tilde{D}$ such that $h^{\prime} T^{\prime}=T^{\prime}$ [MS; Proof of Theorem 3.6]. By [JS; Corollary V.5.1, p. 164], there is a homeomorphism $f: \tilde{D} \rightarrow \tilde{D}$ such that $f$ is isotopic to the identity and such that $f\left(T^{\prime}\right)=T$. We shall denote $f h^{\prime} f^{-1}$ by $h^{\prime}$; hence, $h^{\prime} T=T$.

We claim that $h^{\prime}\left(T_{1} \cup T_{2}\right)=T_{1} \cup T_{2}$. If not, then for some $i$ and $j$ in $\{1,2\}$, we have $h^{\prime} T_{i} \not \subset \partial \tilde{V}$ and $h^{\prime} T_{i} \subset E_{j}(K)$. Hence, $h^{\prime} \tilde{V} \subset E_{j}(K)$, for otherwise, $h^{\prime} \tilde{V}$ contains a characteristic torus (either $T_{1}$ or $T_{2}$ ) in its interior that is not boundary parallel (because of the minimality of $T$ ), which contradicts Lemma 3.1. But if $h^{\prime} \tilde{V} \subset E_{j}(K)$, then $K$ must be a companion of itself, because $\tilde{V}$ is a cable space. Thus, since no knot can be a companion of itself [Sch], we have $h^{\prime}\left(T_{1} \cup T_{2}\right)=T_{1} \cup T_{2}$, and so $h^{\prime} \tilde{V}=\tilde{V}$.

Next we claim that $A^{\prime}\left(=\operatorname{Fix}\left(h^{\prime}\right)\right) \subset \operatorname{Int} \tilde{V}$. If $A^{\prime} \subset \operatorname{Int} E_{i}(K)$, then $h^{\prime} \mid \tilde{V}$ is a free involution. By Theorem 3.6, there is exactly one strong equivalence class of free involutions on $\tilde{V}$, and each involution of this class interchanges the boundary components of $\tilde{V}$. Hence, $h^{\prime} E_{i}(K)=$ $E_{j}(K)(i \neq j)$. This is a contradiction, however, because $\operatorname{Fix}\left(h^{\prime}\right) \subset E_{i}(K)$. On the other hand, if $A^{\prime} \cap\left(T_{1} \cup T_{2}\right) \neq \varnothing$, then $h^{\prime} T_{i}=T_{i}$, for at least one $i \in\{1,2\}$, and $A^{\prime} \cap T_{i}$ contains exactly four points, because $h^{\prime}$ is OP. But then $K$ is strongly invertible, contradicting our hypothesis. Thus $A^{\prime} \subset$ Int $\tilde{V}$. 
Therefore, by Theorem 3.6, there exists a homeomorphism $\varphi: \tilde{V} \rightarrow \tilde{V}$ such that $\varphi$ is isotopic to the identity (on $\tilde{V}$ ) and such that $\varphi\left(h^{\prime} \mid \tilde{V}\right) \varphi^{-1}$ is either $g_{A}, g_{C}$ or $g_{F}$. Since $\varphi$ is isotopic to the identity, we can extend $\varphi$ to $\tilde{D}$. Thus, $\left(\varphi h^{\prime} \varphi^{-1}\right) \mid \tilde{V}$ is either $g_{A}, g_{C}$, or $g_{F}$; we denote $\varphi h^{\prime} \varphi^{-1}$ by $h^{\prime}$. But, as we pointed out in the constructions of $h_{C}$ and $h_{F}$, if $h^{\prime} \mid \tilde{V}$ is either $g_{C}$ or $g_{F}$, then $\tilde{D} / h \nRightarrow S^{3}$, because $\rho \neq 0$ or $K$ has property $P$. Hence, $h^{\prime} \mid \tilde{V}=g_{A}\left(=h_{A} \mid \tilde{V}\right)$.

To see that $D^{\prime}$ and $D(K, \rho)$ belong to the same knot type, define $\alpha$ : $\tilde{D} \rightarrow \tilde{D}$ by $\alpha \mid\left(E_{1}(K) \cup \tilde{V}\right)=$ id and $\alpha\left|E_{2}(K)=\left(h_{A} h^{\prime}\right)\right| E_{2}(K)$ (this is part of Hartley's trick [Ha]). Clearly, $\alpha$ is a well-defined homeomorphism and $h_{A}=\alpha h^{\prime} \alpha^{-1}$. Hence, there exists a homeomorphism $\bar{\alpha}:\left(S^{3}, D^{\prime}\right) \rightarrow$ $\left(S^{3}, D(K, \rho)\right)$, defined by $\bar{\alpha} p^{\prime}=p \alpha$.

Recall that, if $K$ is strongly invertible, then there is an involution $h_{B}$ on $\tilde{D}$ that induces a two-fold covering $p^{\prime}: \tilde{D} \rightarrow S^{3}$ branched over a knot $D^{\prime} ;$ also, $\operatorname{Fix}\left(h_{B}\right)=B$.

Propositron 3.8. If $K$ is a nontrivial, strongly invertible, prime knot, then $D(K, \rho)$ and $D^{\prime}$ belong to distinct knot types; in fact, $D^{\prime}$ is not a doubled knot (Figure 28(b)).

Proof. Since $\tilde{D}$ is irreducible, $D^{\prime}$ is a prime knot. Since $K$ is nontrivial and $\tilde{V}$ is boundary irreducible, the torus $T_{1}\left(=\partial E_{1}(K)\right)$ is incompressible in $\tilde{D}$. Now $T_{1}$ is invariant under $h_{B}$, meets $\operatorname{Fix}\left(h_{B}\right)(=B)$ in exactly four points, and splits $\tilde{D}$ into irreducible, boundary-irreducible parts. It follows from a result of W. B. R. Lickorish, as Bleiler pointed out in [BI], that $D^{\prime}$ is the join of two prime tangles.

On the other hand, Bleiler has shown that any double of a prime knot is not the join of two prime tangles [Bl; Theorem 3.1], and so $D(K, \rho)$ and $D^{\prime}$ belong to distinct knot types. Moreover, if $D^{\prime}$ is a doubled knot (say, $\left.D^{\prime}=D\left(K^{\prime}, \rho\right)\right)$, then $K^{\prime}$ is composite [ $\mathrm{Bl}$; Theorem 3.1].

Thus, assuming that $D^{\prime}=D\left(K^{\prime}, \rho^{\prime}\right)$ (with $K^{\prime}$ composite), we can write

$$
\tilde{D}=E_{1}\left(K^{\prime}\right) \cup_{T_{1}^{\prime}} \tilde{V}^{\prime} \cup_{T_{2}^{\prime}} E_{2}\left(K^{\prime}\right)
$$

with respect to $K^{\prime}$; as usual we have

$$
\tilde{D}=E_{1}(K) \cup_{T_{1}} \tilde{V} \cup_{T_{2}} E_{2}(K) .
$$

Let $\Sigma$ be a characteristic submanifold for $\tilde{D}$. After ambient isotopies of $\tilde{D}$, we can assume (as shown in the Proof of Theorem 3.7) that $\tilde{V}$ is a component $\sigma$ of $\Sigma$ (because $K$ is prime) and that $\tilde{V}^{\prime}$ belongs to a 
component $\sigma^{\prime}$ such that

$$
\sigma^{\prime}=W_{1} \cup_{T_{1}^{\prime}} \tilde{V}^{\prime} \cup_{T_{2}^{\prime}} W_{2},
$$

in which $W_{i}$ is a composing space $(i=1,2)$. Since the number of components of $\partial \sigma^{\prime}$ is $2 n(\geq 4)$ (here $n$ is the number of prime factors of the composite knot $K^{\prime}$ ) and the number of components of $\partial \sigma$ is 2 , we have $\sigma \not \sigma^{\prime} ;$ hence, $\sigma \cap \sigma^{\prime}=\varnothing$. Therefore, $\sigma^{\prime} \subset E_{i}(K)$, for $i=1$ or $i=2$. Consequently, $\sigma^{\prime}$ is.a torus-knot space, a cable space, or a composing space [Ja; Lemma IX.22, p. 188]. But $\sigma^{\prime}$ is obviously neither a torus-knot space nor a cable space. It is not a composing space either, because it has one exceptional fiber and a composing space has none. Thus, we have a contradiction to our assumption that $D^{\prime}$ is a doubled knot.

CoROllary 3.9. If $K$ is a prime knot and if $\rho \neq 0$, then the two-fold branched covering space $\tilde{D}$ of the double $D(K, \rho)$ of $K$ characterizes $D(K, \rho)$ among all doubled knots.

Proof. We begin as we did in the proof of Theorem 3.7. We have $\tilde{D}=E_{1}(K) \cup_{T_{1}} \tilde{V} \cup_{T_{2}} E_{2}(K)$. Let $h_{A}$ be an OP involution on $\tilde{D}$ such that $\operatorname{Fix}\left(h_{A}\right)=A$ (Figure 28) and such that the induced covering $p$ : $\tilde{D} \rightarrow S^{3}$ maps $A$ onto $D(K, \rho)$. Let $h^{\prime}$ be a second OP involution on $\tilde{D}$ with $\operatorname{Fix}\left(h^{\prime}\right)$ a simple closed curve $A^{\prime}$. Moreover, suppose that $h^{\prime}$ induces a covering $p^{\prime}: \tilde{D} \rightarrow S^{3}$ with $p^{\prime}\left(A^{\prime}\right)=D\left(K^{\prime}, \rho^{\prime}\right)$. If $K$ is not strongly invertible, then $D(K, \rho)$ and $D\left(K^{\prime}, \rho^{\prime}\right)$ belong to the same knot type (Theorem 3.7).

So suppose that $K$ is strongly invertible. Then, by an argument analogous to that in the proof of Theorem 3.7, we can vary $h^{\prime}$ so that $h^{\prime}(\tilde{V})=\tilde{V}$ and $h^{\prime} \mid \tilde{V}$ is either $g_{A}$ or $g_{B}$. The proof of Proposition 3.8 shows that, on the one hand, $D\left(K^{\prime}, \rho^{\prime}\right)$ is the join of two prime tangles and $K^{\prime}$ is composite, if $h^{\prime} \mid \tilde{V}=g_{B}$, but that, on the other hand, a contradiction occurs, because $K$ is prime. Hence, $h^{\prime} \mid \tilde{V}=g_{A}$, and the last paragraph of the proof of Theorem 3.7 shows that $D\left(K^{\prime}, \rho^{\prime}\right)$ and $D(K, \rho)$ belong to the same knot type.

Unoriented knots $K_{1}$ and $K_{2}$ in $S^{3}$ belong to the same isotopy type, if there exists an OP homeomorphism $\left(S^{3}, K_{1}\right) \rightarrow\left(S^{3}, K_{2}\right)$. Let $\{K\}$ denote the isotopy type of a knot $K$; let $K^{*}$ denote the mirror image of $K$. With each doubled knot, we associate a pair of integers $(\rho, \eta)$ such that the twisting number $\rho$ is arbitrary and the self-intersection number $\eta$ belongs to $\{-2,2\}$. The triple $(\{K\}, \rho, \eta)$ characterizes $\{D(K ; \rho, \eta)\}$, the isotopy 
type of the $(\rho, \eta)$-double of $K$; moreover, $\{D(K ; \rho, \eta)\}$ completely determines the triple $(\{K\}, \rho, \eta)$ except in two cases when $K$ is trivial [Sch; p. 234] (cf. [Wh $; \mathbf{W h}_{\mathbf{1}} ;$ p. 261]). Note that the mirror image of $D(K ; \rho, \eta)$ is $D\left(K^{*},-\rho,-\eta\right)$. Our last result follows from Theorem 2.1 of $\left[\mathbf{W h}_{\mathbf{1}}\right]$ and from Corollary 3.9.

Corollary 3.10. Let $K$ and $K^{\prime}$ be knots in $S^{3}$, and suppose that $K$ is prime. Let $\rho$ and $\eta$ be fixed integers; $\rho \neq 0$, but otherwise arbitrary; $\eta= \pm 2$. Finally, let $\tilde{D}, \tilde{D}^{\prime}$ and $\tilde{D}_{0}^{\prime}$ denote the two-fold branched covering spaces of $D(K ; \rho, \eta), D\left(K^{\prime} ; \rho, \eta\right)$, and $D\left(K^{\prime *} ; \rho, \eta\right)$, respectively. Then $K$ and $K^{\prime}$ belong to the same knot type if and only if either $\tilde{D} \cong \tilde{D}^{\prime}$ or $\tilde{D} \cong \tilde{D}_{0}^{\prime}$.

Proof. Necessity. Suppose that $K$ and $K^{\prime}$ belong to the same knot type. Then, by $\left[\mathbf{W h}_{\mathbf{1}} ;\right.$ Theorem 2.1 , p. 263], either $E(D(K ; \rho, \eta)) \cong$ $E\left(D\left(K^{\prime} ; \rho, \eta\right)\right)$ or $E(D(K ; \rho, \eta)) \cong E\left(D\left(K^{\prime *} ; \rho, \eta\right)\right)$. Hence, either $D(K ; \rho, \eta)$ and $D\left(K^{\prime} ; \rho, \eta\right)$ or $D(K ; \rho, \eta)$ and $D\left(K^{\prime *} ; \rho, \eta\right)$ belong to the same knot type $([\mathbf{G}],[\mathbf{B M}])$. But then, either $\tilde{D} \cong \tilde{D}^{\prime}$ or $\tilde{D} \cong \tilde{D}_{0}^{\prime}$, as required.

Sufficiency. Suppose that $\tilde{D} \cong \tilde{D}^{\prime}$ or $\tilde{D} \cong \tilde{D}_{0}^{\prime}$. By Corollary 3.9 , either $D(K ; \rho, \eta)$ and $D\left(K^{\prime} ; \rho, \eta\right)$ or $D(K ; \rho, \eta)$ and $D\left(K^{\prime *} ; \rho, \eta\right)$ belong to the same knot type. Hence either $\{D(K ; \rho, \eta)\}=\left\{D\left(K^{\prime} ; \rho, \eta\right)\right\}$, $\{D(K ; \rho, \eta)\}=\left\{D\left(K^{\prime *} ; \rho, \eta\right)\right\},\{D(K ; \rho, \eta)\}=\left\{D\left(K^{\prime *} ;-\rho,-\eta\right)\right\}$, or $\{D(K ; \rho, \eta)\}=\left\{D\left(K^{\prime} ;-\rho,-\eta\right)\right\}$. But neither of the last two possibilities can hold, because $K$ is nontrivial and $\eta= \pm 2$ (cf. the proof of Theorem 2.1 of $\left.\left[\mathbf{W h}_{1}\right]\right)$. Therefore, either $\{K\}=\left\{K^{\prime}\right\}$ or $\{K\}=\left\{K^{\prime *}\right\}$ (see the paragraph preceding the statement of this corollary).

\section{REFERENCES}

[BGM] J. Birman, F. González-Acuña and J. M. Montesinos, Heegaard splittings of prime 3-manifolds are not unique, Michigan Math. J., 23 (1976), 97-103.

[B1] Steven A. Bleiler, Knots prime on many strings, Trans. Amer. Math. Soc., (to appear).

[BoGM] M. Boileau, F. González-Acuña and J. M. Montesinos, Surgery on double knots and symmetries, (to appear).

[BM] R. H. Bing and J. M. Martin, Cubes with knotted holes, Trans. Amer. Math. Soc., 155 (1971), 217-231.

[BuM] G. Burde and K. Murasugi, Links and Seifert fiber spaces, Duke Math. J., 37 (1970), 89-93.

[FS] S. Furusawa and M. Sakuma, Dehn surgery on symmetric knots, Math. Sem. Notes, 11 (1983), 179-198. 
[G] F. Conzález-Acuña, Ph.D. Thesis, Princeton University, 1969.

[GH] C. McA. Gordon and Wolfgang Heil, Cyclic normal subgroups of fundamental groups of 3-manifolds, Topology, 14 (1975), 305-309.

[GL] C. McA. Gordon and R. A. Litherland, Incompressible surfaces in branched coverings, Proceedings of the Symposium on the Smith Conjecture, Columbia University, (1978), 139-152.

[Ha] R. Hartley, Knots and involutions, Math. Zeit., 171 (1980), 175-185.

[Ho] C. D. Hodgson, Involutions and isotopies of lens spaces, MS Thesis, University of Melbourne, 1981.

[Ha] W. Jaco, Lectures on Three-Manifold Topology, Regional Conference Series 43, Amer. Math. Soc., Providence, R. I., 1980.

[JS] W. Jaco and P. Shalen, Seifert Fibered Spaces in 3-Manifolds, Memoirs of the Amer. Math. Soc., Vol. 21, No. 220, Amer. Math. Soc., Providence, R. I., 1979.

[Ki] P. K. Kim, Involutions on Klein spaces $M(p, q)$, Trans. Amer. Math. Soc., 268 (1981), 377-409.

[KT $\mathrm{KT}_{1}$ P. K. Kim and J. L. Tollefson, PL involutions of fibered 3-manifolds, Trans. Amer. Math. Soc., 232 (1977), 221-237.

$\left[\mathrm{KT}_{2}\right] \quad$ Splitting the PL involutions of non-prime 3-manifolds, Michigan Math. J., 27 (1980), 259-274.

[KwT] K. W. Kwun and J. L. Tollefson, Extending PL involutions of a compact manifold, Amer. J. Math., 99 (1977), 995-1001.

[M] José M. Montesinos, Variedades de Seifert que son recubridores ciclicos ramificados de dos hojas, Bol. Soc. Math. Mexicana (2), 18 (1973), 1-32.

$\left[\mathrm{Mo}_{1}\right] \quad$, Surgery on links and double branched covers, Knots, Groups, and 3-Manifolds, Ann. of Math. Studies, 84, 227-259, Princeton University Press, Princeton, N. J., 1975.

$\left[\mathrm{Mo}_{2}\right] \quad$ Sobre la representacion de variedades tridimensionales, unpublished preprint (1975).

[MS] W. H. Meeks and P. Scott, Finite group actions on 3-manifolds, preprint.

[MW] José M. Montesinos and W. Whitten, Constructions of two-fold branched covering spaces, Abstracts Amer. Math. Soc., 4 (Mar., 1983), Abstract 802-57-11, p. 178.

[Ru] J. H. Rubinstein, Representations of some 3-manifolds as 2-fold cyclic branched covers of $S^{3}$, Notices Amer. Math. Soc., 25 (1978), Abstract 78T-G7, A-18.

[Sa] M. Sakuma, Surface bundles over $S^{1}$ which are 2-fold branched cyclic coverings of $S^{3}$, Math. Sem. notes, 9 (1981), 159-180.

[Sch] H. Schubert, Knoten und Vollringe, Acta Math., 90 (1953), 131-286.

[Ta] M. Takahashi, Two knots with the same two fold branched covering space, Yokohama Math. J., 25 (1977), 91-99.

$\left[\mathrm{Ta}_{1}\right] \quad \ldots$, On homology spheres obtained by surgery on the figure-eight knot, Proc. Sympos. Res. Inst. Math. Sci. Univ. Kyoto, 309 (1977) (in Japanese).

[Th] W. Thurston, The Geometry and Topology of 3-Manifolds, Lecture notes, Princeton University, 1978-1979.

[Tol ${ }_{1}$ J. L. Tollefson, involutions on $S^{1} \times S^{2}$ and other 3-manifolds, Trans. Amer. Math. Soc., 183 (1973), 139-152.

[ $\mathrm{Tol}_{2}$ ] _ Involutions of Seifert fiber spaces, Pacific J. Math., 74 (1978), 519-529.

[ $\left.\mathrm{Tol}_{3}\right]$ _ Involutions of sufficiently large 3-manifolds, Topoloy, 20 (1981), 323-352.

[Wa $\left.\mathrm{W}_{1}\right] \mathrm{F}$. Waldhausen, Eine Klasse von 3-dimensionalen Mannigfaltigkeiten I, II, Invent. Math., 3 (1967), 308-333; and Invent. Math., 4 (1967), 87-117.

[Wa $\left.\mathrm{W}_{2}\right]$, Uber Involutionen der 3-Sphäre, Topology, 8 (1969), 81-91. 
[Wh $\mathrm{W}_{1}$ ] W. Whitten, Algebraic and geometric characterizations of knots, Invent. Math., 26 (1974), 259-270.

[Wh $\mathrm{Wh}_{2}$, Inverting double knots, Pacific J. Math., 97 (1981), 209-216.

$\left[\mathrm{Ze}_{1}\right] \mathrm{H}$. Zieschang, On extensions of fundamental groups of surfaces and related groups, Bull. Amer. Math. Soc., 77 (1971), 1116-1119.

$\left[\mathrm{Ze}_{2}\right]$, Addendum to: On extensions of fundamental groups of surfaces and related groups, Bull. Amer. Math. Soc., 80 (1974), 366-367.

Received July 26, 1983 and in revised form June 25, 1985. The second author wishes to thank the Institute for Advanced Study, Princeton, N. J. for its hospitality while part of this work was done. The first author was supported by "Comité Conjunto HispanoNorteamericano" and NSF Grant 8120790.

UNIVERSIDAD DE ZARAGOZA AND

MATHEMATICAL SCIENCES RESEARCH INSTITUTE

AND

UNIVERSITY OF SOUTHWESTERN LOUISIANA

LAFAYETTE, LA 70504 


\title{
PACIFIC JOURNAL OF MATHEMATICS \\ EDITORS
}

\author{
V. S. VARADARAJAN \\ (Managing Editor) \\ University of California \\ Los Angeles, CA 90024 \\ HERBERT CLEMENS \\ University of Utah \\ Salt Lake City, UT 84112 \\ R. FINN \\ Stanford University \\ Stanford, CA 94305
}

\author{
HERMANN FLASCHKA \\ University of Arizona \\ Tucson, AZ 85721
}

RAMESh A. GANGOLLI

University of Washington Seattle, WA 98195

VAUGHAN F. R. JONES

University of California

Berkeley, CA 94720

ROBION KIRBY

University of California

Berkeley, CA 94720
C. C. MOORE

University of California

Berkeley, CA 94720

H. SAMELSON

Stanford University

Stanford, CA 94305

HAROLD STARK

University of California, San Diego

La Jolla, CA 92093

\section{ASSOCIATE EDITORS}

\author{
R. ARENS \\ E. F. BECKENBACH \\ B. H. NeUmanN \\ F. WOLF \\ K. YOSHIDA \\ (1906-1982)

\section{SUPPORTING INSTITUTIONS} \\ UNIVERSITY OF ARIZONA \\ UNIVERSITY OF BRITISH COLUMBIA \\ CALIFORNIA INSTITUTE OF TECHNOLOGY \\ UNIVERSITY OF CALIFORNIA \\ MONTANA STATE UNIVERSITY \\ UNIVERSITY OF NEVADA, RENO \\ NEW MEXICO STATE UNIVERSITY \\ OREGON STATE UNIVERSITY \\ UNIVERSITY OF OREGON \\ UNIVERSITY OF SOUTHERN CALIFORNIA \\ STANFORD UNIVERSITY \\ UNIVERSITY OF HAWAII \\ UNIVERSITY OF TOKYO \\ UNIVERSITY OF UTAH \\ WASHINGTON STATE UNIVERSITY \\ UNIVERSITY OF WASHINGTON
}

The Supporting Institutions listed above contribute to the cost of publication of this Journal, but they are not owners or publishers and have no responsibility for its content or policies.

Mathematical papers intended for publication in the Pacific Journal of Mathematics should be in typed form or offset-reproduced (not dittoed), double spaced with large margins. Please do not use built up fractions in the text of the manuscript. However, you may use them in the displayed equations. Underline Greek letters in red, German in green, and script in blue. The first paragraph must be capable of being used separately as a synopsis of the entire paper. In particular it should contain no bibliographic references. Please propose a heading for the odd numbered pages of less than 35 characters. Manuscripts, in triplicate, may be sent to any one of the editors. Please classify according to the scheme of Math. Reviews, Index to Vol. 39. Supply name and address of author to whom proofs should be sent. All other communications should be addressed to the managing editor, or Elaine Barth, University of California, Los Angeles, California 90024.

There are page-charges associated with articles appearing in the Pacific Journal of Mathematics. These charges are expected to be paid by the author's University, Government Agency or Company. If the author or authors do not have access to such Institutional support these charges are waived. Single authors will receive 50 free reprints; joint authors will receive a total of 100 free reprints. Additional copies may be obtained at cost in multiples of 50 .

The Pacific Journal of Mathematics is issued monthly as of January 1966. Regular subscription rate: $\$ 190.00$ a year (5 Vols., 10 issues). Special rate: $\$ 95.00$ a year to individual members of supporting institutions.

Subscriptions, orders for numbers issued in the last three calendar years, and changes of address should be sent to Pacific Journal of Mathematics, P.O. Box 969, Carmel Valley, CA 93924, U.S.A. Old back numbers obtainable from Kraus Periodicals Co., Route 100, Millwood, NY 10546.

The Pacific Journal of Mathematics at P.O. Box 969, Carmel Valley, CA 93924 (ISSN 0030-8730) publish'es 5 volumes per year. Application to mail at Second-class postage rates is pending at Carmel Valley, California, and additional mailing offices. Postmaster: send address changes to Pacific Journal of Mathematics, P.O. Box 969, Carmel Valley, CA 93924.

PUBLISHED BY PACIFIC JOURNAL OF MATHEMATICS, A NON-PROFIT CORPORATION

Copyright (C) 1986 by Pacific Journal of Mathematics 


\section{Pacific Journal of Mathematics}

\section{Vol. 125, No. $2 \quad$ October, 1986}

Dale Edward Alspach, On $\mathscr{L}_{p, \lambda}$ spaces for small $\lambda \ldots \ldots \ldots \ldots \ldots \ldots 257$

Jong Sook Bae and Sangsuk Yie, Range of Gateaux differentiable operators

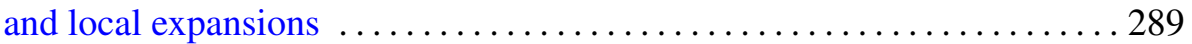

Hubert Berens and Lothar Hetzelt, On accretive operators on $l_{n}^{\infty} \ldots \ldots 301$

Marie-Françoise Bidaut-Véron, Global existence and uniqueness results for singular solutions of the capillarity equation ................. 317

Donald M. Davis and Mark Mahowald, Classification of the stable homotopy types of stunted real projective spaces ................. 335

Aad Dijksma, Heinz K. Langer and Hendrik S. V. de Snoo, Unitary

colligations in $\Pi_{\kappa}$-spaces, characteristic functions and Štraus extensions

Michel Enock and Jean-Marie Schwartz, Algèbres de Kac

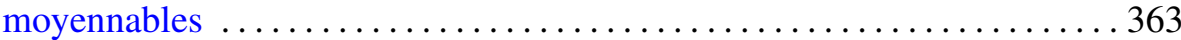

Seppo Granlund, Peter Lindqvist and Olli Martio, Note on the

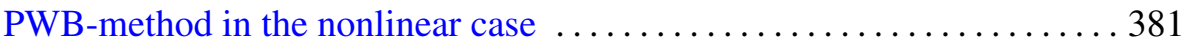

Palle E. T. Jorgensen, Analytic continuation of local representations of Lie

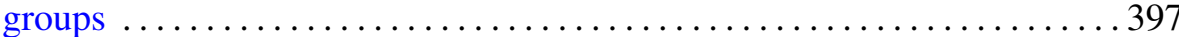

Robert P. Kaufman, Plane curves and removable sets ................409

José M. Montesinos and Wilbur Carrington Whitten, Constructions of two-fold branched covering spaces $\ldots \ldots \ldots \ldots \ldots$.

Benedict Seifert, Highly transitive group actions on trees and normalizing Tits systems

Charles Stuart Stanton, Counting functions and majorization for Jensen

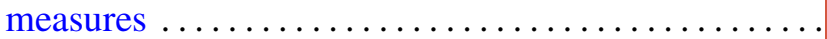

Luen-Fai Tam, On existence criteria for capillary free surfaces without gravity

Zhuocheng Yang, Exposed points of left invariant means 